\title{
C-C Bond Formation Catalyzed Heterogeneously by Nickel-on-Graphite $\left(\mathrm{Ni} / \mathrm{C}_{\mathrm{g}}\right)$
}

\author{
Bruce H. Lipshutz, ${ }^{*}$ Tom Butler, and Elizabeth Swift \\ Department of Chemistry and Biochemistry \\ University of California \\ Santa Barbara, California 93106 USA
}

Supporting Information 


\section{Standard $\mathrm{Ni} / \mathrm{C}_{\mathrm{g}}$ catalyzed cross-couplings of boronic acids with aryl halides using conventional}

heating. To a flame dried, argon purged $25 \mathrm{~mL}$ round bottom flask were added Ni/ $\mathrm{C}_{\mathrm{g}}(96.0 \mathrm{mg}, 0.08$ mmol) and $\mathrm{PPh}_{3}(63.0 \mathrm{mg}, 0.24 \mathrm{mmol})$ under argon at rt. THF (2 mL) was then added followed by $n$ $\operatorname{BuLi}(63 \mu \mathrm{L}, 2.55 \mathrm{M}$ in hexanes, $0.16 \mathrm{mmol})$. The solution was allowed to stir at $\mathrm{rt}$ for $15 \mathrm{~min}$. The aryl halide (1.00 mmol), boronic acid (2.00 mmol), KF (174 mg, $3.00 \mathrm{mmol})$, LiOH (120 mg, $5.00 \mathrm{mmol})$ and dioxane $(2 \mathrm{~mL})$ were then added to the reaction mixture. The flask was loaded into a preheated oil bath and warmed to reflux. The reaction was allowed to stir until TLC indicated complete disappearance of the starting material. After cooling to $\mathrm{rt}$, the reaction mixture was filtered and extracted with $\mathrm{Et}_{2} \mathrm{O}(3 \times 10 \mathrm{~mL})$ and $\mathrm{H}_{2} \mathrm{O}(3 \times 10 \mathrm{~mL})$. The combined organics were washed with brine and dried over anhydrous $\mathrm{MgSO}_{4}$. The solvent was removed in vacuo and the crude product was purified by silica gel chromatography (3:1 hex/ EtOAc).

4-Methoxybiphenyl (Table 1, entry A). Using the standard procedure outlined above, the following amounts of reagents were used: $\mathrm{Ni} / \mathrm{C}(98.0 \mathrm{mg}, 0.08 \mathrm{mmol}), \mathrm{PPh}_{3}(63.0 \mathrm{mg}, 0.24 \mathrm{mmol}), \mathrm{THF}(2 \mathrm{~mL})$, $n$-BuLi $(63 \mu \mathrm{L}, 2.55 \mathrm{M}$ in hexanes, $0.16 \mathrm{mmol})$, 4-chloroanisole $(0.127 \mathrm{~mL}, 1.00 \mathrm{mmol})$, phenylboronic acid (244 mg, $2.00 \mathrm{mmol}), \mathrm{KF}$ (174 mg, $3.00 \mathrm{mmol}), \mathrm{LiOH}(120 \mathrm{mg}, 5.00 \mathrm{mmol})$ and dioxane $(2 \mathrm{~mL})$. The reaction was allowed to stir overnight at which point TLC indicated incomplete conversion of the starting material. After cooling to rt, the reaction mixture was filtered and extracted with $\mathrm{Et}_{2} \mathrm{O}(3 \times 10$ $\mathrm{mL})$ and $\mathrm{H}_{2} \mathrm{O}(3 \times 10 \mathrm{~mL})$. The combined organics were washed with brine and dried over anhydrous $\mathrm{MgSO}_{4}$. The solvent was removed in vacuo and the crude product was purified by silica gel chromatography (3:1 hex/ EtOAc). After purification $74 \mathrm{mg}$ of the title compound was isolated (40\%). $\mathrm{R}_{\mathrm{f}}=0.75$ (3:1 hex/ EtOAc). Spectral data of the product matched that of previously reported spectra. ${ }^{1}$ 3,4',4-Trimethoxybiphenyl (Table 1, entry B; using Ni/C). Using the standard procedure outlined above, the following amounts of reagents were used: $\mathrm{Ni} / \mathrm{C}(98.0 \mathrm{mg}, 0.08 \mathrm{mmol}), \mathrm{PPh}_{3}(63.0 \mathrm{mg}, 0.24$ mmol), THF (2 mL), $n$-BuLi $(63 \mu \mathrm{L}, 2.55 \mathrm{M}$ in hexanes, $0.16 \mathrm{mmol})$, 4-chloroanisole $(0.127 \mathrm{~mL}, 1.00$ mmol), 3,4-dimethoxyphenylboronic acid (244 mg, $2.00 \mathrm{mmol}), \mathrm{KF}$ (174 mg, $3.00 \mathrm{mmol})$, LiOH (120 
$\mathrm{mg}, 5.00 \mathrm{mmol})$ and dioxane $(2 \mathrm{~mL})$. The reaction was allowed to stir for $14 \mathrm{~h}$ at which point TLC indicated complete disappearance of the starting material. After cooling to $\mathrm{rt}$, the reaction mixture was filtered and extracted with $\mathrm{Et}_{2} \mathrm{O}(3 \times 10 \mathrm{~mL})$ and $\mathrm{H}_{2} \mathrm{O}(3 \times 10 \mathrm{~mL})$. The combined organics were washed with brine and dried over anhydrous $\mathrm{MgSO}_{4}$. The solvent was removed in vacuo and the crude product was purified by silica gel chromatography (3:1 hex/EtOAc). After purification $221 \mathrm{mg}$ of the title compound was isolated $(90 \%) . \mathrm{R}_{\mathrm{f}}=0.33$ (3:1 hex/EtOAc). Spectra matched entry $\mathrm{B}, \mathrm{Ni} / \mathrm{C}_{\mathrm{g}}$.

3,4',4-Trimethoxybiphenyl (Table 1, entry $\mathrm{B}$; using $\mathrm{Ni} / \mathrm{C}_{\mathrm{g}}$ ). Using the standard procedure outlined above, the following amounts of reagents were used: $\mathrm{Ni} / \mathrm{C}_{\mathrm{g}}(96.0 \mathrm{mg}, 0.08 \mathrm{mmol}), \mathrm{PPh}_{3}(63.0 \mathrm{mg}, 0.24$ mmol), THF (2 mL), $n$-BuLi $(63 \mu \mathrm{L}, 2.55 \mathrm{M}$ in hexanes, $0.16 \mathrm{mmol})$, 4-chloroanisole $(0.127 \mathrm{~mL}, 1.00$ mmol), 3,4-dimethoxyphenylboronic acid (244 mg, $2.00 \mathrm{mmol}), \mathrm{KF}$ (174 mg, $3.00 \mathrm{mmol})$, LiOH (120 $\mathrm{mg}, 5.00 \mathrm{mmol})$ and dioxane $(2 \mathrm{~mL})$. The reaction was allowed to stir for $10 \mathrm{~h}$ at which point TLC indicated complete disappearance of the starting material. After cooling to rt, the reaction mixture was filtered and extracted with $\mathrm{Et}_{2} \mathrm{O}(3 \times 10 \mathrm{~mL})$ and $\mathrm{H}_{2} \mathrm{O}(3 \times 10 \mathrm{~mL})$. The combined organics were washed with brine and dried over anhydrous $\mathrm{MgSO}_{4}$. The solvent was removed in vacuo and the crude product was purified by silica gel chromatography (3:1 hex/EtOAc). After purification $223 \mathrm{mg}$ of the title compound was isolated $(92 \%) . \mathrm{R}_{\mathrm{f}}=0.33\left(3: 1\right.$ hex/EtOAc); ${ }^{1} \mathrm{H} \mathrm{NMR}\left(\mathrm{CDCl}_{3}, 400 \mathrm{MHz}\right) \delta 7.35(\mathrm{t}, J=$ $7.9,1 \mathrm{H}), 7.15(\mathrm{~m}, 2 \mathrm{H}), 7.11(\mathrm{~m}, 2 \mathrm{H}), 6.94(\mathrm{~d}, J=8.3,1 \mathrm{H}), 6.87(\mathrm{~m}, 1 \mathrm{H}), 3.95(\mathrm{~s}, 3 \mathrm{H}), 3.93(\mathrm{~s}, 1 \mathrm{H}), 3.87$ $(\mathrm{s}, 1 \mathrm{H}) ;{ }^{13} \mathrm{C} \mathrm{NMR}\left(\mathrm{CDCl}_{3}, 100 \mathrm{MHz}\right) \delta 160.1,149.2,148.8,142.8,134.2,129.9,119.6,113.0,112.1$, 111.5, 110.6, 56.1, 55.5; HREIMS $m / z$ calcd for $\mathrm{C}_{15} \mathrm{H}_{16} \mathrm{O}_{3}$ 244.1099; found 244.1097.

2-Methoxybiphenyl (Table 1, entry C; using Ni/C). Using the standard procedure outlined above, the following amounts of reagents were used: $\mathrm{Ni} / \mathrm{C}(98.0 \mathrm{mg}, 0.08 \mathrm{mmol}), \mathrm{PPh}_{3}(63.0 \mathrm{mg}, 0.240 \mathrm{mmol})$, THF (2 mL), $n$-BuLi $(63 \mu \mathrm{L}, 2.55 \mathrm{M}$ in hexanes, $0.16 \mathrm{mmol}), 2$-chloroanisole $(0.127 \mathrm{~mL}, 1.00 \mathrm{mmol})$, phenylboronic acid (244 mg, $2.00 \mathrm{mmol}), \mathrm{KF}(174 \mathrm{mg}, 3.00 \mathrm{mmol}), \mathrm{LiOH}(120 \mathrm{mg}, 5.00 \mathrm{mmol})$ and dioxane $(2 \mathrm{~mL})$. The reaction was allowed to stir overnight at which point TLC indicated no consumption of the starting material. ${ }^{1}$ 
2-Methoxybiphenyl (Table 1 , entry $\mathrm{C}$; using $\mathrm{Ni} / \mathrm{C}_{\mathrm{g}}+$ conventional heating). Using the standard procedure outlined above, the following amounts of reagents were used: $\mathrm{Ni} / \mathrm{C}_{\mathrm{g}}(96.0 \mathrm{mg}, 0.08 \mathrm{mmol})$, $\mathrm{PPh}_{3}(63.0 \mathrm{mg}, 0.24 \mathrm{mmol})$, THF $(2 \mathrm{~mL}), n$-BuLi $(63 \mu \mathrm{L}, 2.55 \mathrm{M}$ in hexanes, $0.16 \mathrm{mmol}), 2$ chloroanisole $(0.127 \mathrm{~mL}, 1.00 \mathrm{mmol})$, phenylboronic acid $(244 \mathrm{mg}, 2.00 \mathrm{mmol}), \mathrm{KF}(174 \mathrm{mg}, 3.00$ $\mathrm{mmol})$, $\mathrm{LiOH}(120 \mathrm{mg}, 5.00 \mathrm{mmol})$ and dioxane $(2 \mathrm{~mL})$. The reaction was allowed to stir for $12 \mathrm{~h}$ at which point TLC indicated complete disappearance of the starting material. After cooling to $\mathrm{rt}$, the reaction mixture was filtered and extracted with $\mathrm{Et}_{2} \mathrm{O}(3 \times 10 \mathrm{~mL})$ and $\mathrm{H}_{2} \mathrm{O}(3 \times 10 \mathrm{~mL})$. The combined organics were washed with brine and dried over anhydrous $\mathrm{MgSO}_{4}$. The solvent was removed in vacuo and the crude product was purified by silica gel chromatography (3:1 hex/EtOAc). After purification $164 \mathrm{mg}$ of the title compound was isolated $(89 \%) . \mathrm{R}_{\mathrm{f}}=0.75$ (3:1 hex/EtOAc). Spectral data of the product matched that of previously reported spectra. ${ }^{1}$

Representative Procedure for the Microwave-Assisted Nickel-on-Graphite Catalyzed Suzuki Coupling. $\mathrm{Ni} / \mathrm{C}_{\mathrm{g}}$ catalyzed cross-coupling of a boronic acid with an aryl halide; 2-methoxybipenyl (Table 1, entry C). To a flame dried, argon purged Emrys Optimizer 2-5 mL pyrex reaction vessel were added $\mathrm{Ni} / \mathrm{C}_{\mathrm{g}}(96.0 \mathrm{mg}, 0.08 \mathrm{mmol})$ and $\mathrm{PPh}_{3}(63.0 \mathrm{mg}, 0.24 \mathrm{mmol})$ under argon at rt. THF $(2 \mathrm{~mL})$ was then added followed by $n$-BuLi $(63 \mu \mathrm{L}, 2.55 \mathrm{M}$ in hexanes, $0.16 \mathrm{mmol})$. The solution was allowed to stir at $\mathrm{rt}$ for $15 \mathrm{~min}$. 2-Chloroanisole $(0.127 \mathrm{~mL}, 1.00 \mathrm{mmol})$, phenylboronic acid (244 mg, $2.00 \mathrm{mmol})$, KF (174 mg, $3.00 \mathrm{mmol}), \mathrm{LiOH}(120 \mathrm{mg}, 5.00 \mathrm{mmol})$ and dioxane $(2 \mathrm{~mL})$ were then added to the reaction mixture. The reaction vessel was placed in the Emrys Optimizer using the following specifications: Temperature: $200{ }^{\circ} \mathrm{C}$, Time: 2700 sec., Fixed Hold Time: On, Sample Absorption: Normal, Pre-stirring: $45 \mathrm{sec}$. After cooling to rt, the reaction mixture was filtered and extracted with $\mathrm{Et}_{2} \mathrm{O}(3 \times 10 \mathrm{~mL})$ and $\mathrm{H}_{2} \mathrm{O}(3 \times 10 \mathrm{~mL})$. The combined organics were washed with brine and dried over anhydrous $\mathrm{MgSO}_{4}$. The solvent was removed in vacuo and the crude product was purified by silica gel chromatography (3:1 hex/EtOAc). After purification $161 \mathrm{mg}$ of the title compound was isolated (87\%). $\mathrm{R}_{\mathrm{f}}=0.75(3: 1 \mathrm{hex} / \mathrm{EtOAc})$. 
2,3',4'-Trimethoxybiphenyl (Table 1, entry D). Using the standard procedure outlined above, the following amounts of reagents were used: $\mathrm{Ni} / \mathrm{C}_{\mathrm{g}}(48.0 \mathrm{mg}, 0.04 \mathrm{mmol}), \mathrm{PPh}_{3}(31.5 \mathrm{mg}, 0.120 \mathrm{mmol})$, THF (1 mL), $n$-BuLi $(32 \mu \mathrm{L}, 2.55 \mathrm{M}$ in hexanes, $0.08 \mathrm{mmol}), 2$-chloroanisole (0.64 $\mathrm{mL}, 0.50 \mathrm{mmol})$, 3,4-dimethoxyphenylboronic acid (182 mg, $1.50 \mathrm{mmol})$, KF (87.0 mg, $1.50 \mathrm{mmol})$, LiOH (60.0 mg, $2.50 \mathrm{mmol})$, and dioxane $(1.5 \mathrm{~mL})$. Mircowave conditions: Temperature: $200{ }^{\circ} \mathrm{C}$, Time: 2700 sec., Fixed Hold Time: On, Sample Absorption: Normal, Pre-stirring: $45 \mathrm{sec}$. After purification $81 \mathrm{mg}$ of the title compound was isolated $(87 \%) . \mathrm{R}_{\mathrm{f}}=0.33(3: 1$ hex/EtOAc). Spectral data on the product matched that of previously reported spectra. ${ }^{1}$

4-Methylbiphenyl (Table 1, entry E). Using the standard conventional procedure outlined above, the following amounts of reagents were used: $\mathrm{Ni} / \mathrm{C}_{\mathrm{g}}(96.0 \mathrm{mg}, 0.08 \mathrm{mmol}), \mathrm{PPh}_{3}(63.0 \mathrm{mg}, 0.240 \mathrm{mmol})$, THF (2 mL), $n$-BuLi $(63 \mu \mathrm{L}, 2.55 \mathrm{M}$ in hexanes, $0.16 \mathrm{mmol}), 4$-bromotoluene (171 $\mathrm{mg}, 1.00 \mathrm{mmol})$, phenylboronic acid (244 mg, $2.00 \mathrm{mmol}), \mathrm{KF}$ (174 mg, $3.00 \mathrm{mmol}), \mathrm{LiOH}(120 \mathrm{mg}, 5.00 \mathrm{mmol})$ and dioxane $(2 \mathrm{~mL})$. The reaction was allowed to stir for $9 \mathrm{~h}$ at which point TLC indicated complete disappearance of the starting material. After cooling to $\mathrm{rt}$, the reaction mixture was filtered and extracted with $\mathrm{Et}_{2} \mathrm{O}(3 \times 10 \mathrm{~mL})$ and $\mathrm{H}_{2} \mathrm{O}(3 \times 10 \mathrm{~mL})$. The combined organics were washed with brine and dried over anhydrous $\mathrm{MgSO}_{4}$. The solvent was removed in vacuo and the crude product was purified by silica gel chromatography (3:1 hex/EtOAc). After purification $146 \mathrm{mg}$ of the title compound was isolated (87\%). $R_{\mathrm{f}}=0.79(3: 1 \mathrm{hex} / \mathrm{EtOAc})$. Spectral data of the product matched that of previously reported spectra. ${ }^{1}$

3,4-Dimethoxy-4'-methylbiphenyl (Table 1, entry F). Using the standard conventional procedure outlined above, the following amounts of reagents were used: $\mathrm{Ni} / \mathrm{C}_{\mathrm{g}}(96.0 \mathrm{mg}, 0.08 \mathrm{mmol}), \mathrm{PPh}_{3}(63.0$ mg, $0.240 \mathrm{mmol})$, THF (2 mL), $n$-BuLi (63 $\mu \mathrm{L}, 2.55 \mathrm{M}$ in hexanes, $0.16 \mathrm{mmol})$, ), 4-bromotoluene (171 mg, $1.00 \mathrm{mmol}$ ), 3,4-dimethoxyphenylboronic acid (244 mg, $2.00 \mathrm{mmol})$, KF (174 mg, $3.00 \mathrm{mmol})$, $\mathrm{LiOH}(120 \mathrm{mg}, 5.00 \mathrm{mmol})$ and dioxane $(2 \mathrm{~mL})$. The reaction was allowed to stir for $7 \mathrm{~h}$ at which point TLC indicated complete disappearance of the starting material. After cooling to $\mathrm{rt}$, the reaction mixture 
was filtered and extracted with $\mathrm{Et}_{2} \mathrm{O}(3 \times 10 \mathrm{~mL})$ and $\mathrm{H}_{2} \mathrm{O}(3 \times 10 \mathrm{~mL})$. The combined organics were washed with brine and dried over anhydrous $\mathrm{MgSO}_{4}$. The solvent was removed in vacuo and the crude product was purified by silica gel chromatography (3:1 hex/EtOAc). After purification $196 \mathrm{mg}$ of the title compound was isolated $(86 \%) . \mathrm{R}_{\mathrm{f}}=0.35\left(3: 1\right.$ hex/EtOAc). ${ }^{1} \mathrm{H} \mathrm{NMR}\left(\mathrm{CDCl}_{3}, 400 \mathrm{MHz}\right) \delta 7.49(\mathrm{~m}$, 2H), $7.25(\mathrm{~m}, 2 \mathrm{H}), 7.20(\mathrm{~m}, 1 \mathrm{H}), 7.13(\mathrm{~m}, 2 \mathrm{H}), 6.90(\mathrm{~m}, 1 \mathrm{H}), 3.97(\mathrm{~s}, 3 \mathrm{H}), 3.92(\mathrm{~s}, 3 \mathrm{H}), 2.40(\mathrm{~s}, 3 \mathrm{H}) ;{ }^{13} \mathrm{C}$ $\mathrm{NMR}\left(\mathrm{CDCl}_{3}, 100 \mathrm{MHz}\right) \delta 149.3,148.8,138.4,136.7,134.4,129.6,126.9,119.3,111.6,110.4,56.1$, 21.2; HREIMS $m / z$ calcd for $\mathrm{C}_{15} \mathrm{H}_{16} \mathrm{O}_{2} 228.1150$; found 228.1156 .

Standard $\mathrm{Ni} / \mathrm{C}_{\mathrm{g}}$ catalyzed cross-couplings of boronic acids with aryl tosylates. To an Emrys Optimizer 2-5 $\mathrm{mL}$ pyrex reaction vessel was added $\mathrm{Ni} / \mathrm{C}_{\mathrm{g}}(96.0 \mathrm{mg}, 0.08 \mathrm{mmol})$ and $\mathrm{PPh}_{3}(63.0 \mathrm{mg}$, $0.240 \mathrm{mmol})$ under argon at rt. THF $(2 \mathrm{~mL})$ was added followed by $n$-BuLi $(63 \mu \mathrm{L}, 2.55 \mathrm{M}$ in hexanes, $0.16 \mathrm{mmol})$. The solution was allowed to stir at $\mathrm{rt}$ for $15 \mathrm{~min}$. The aryl tosylate $(1.00 \mathrm{mmol})$, boronic acid (2.00 mmol), KF (174 mg, $3.00 \mathrm{mmol}), \mathrm{LiOH}(120 \mathrm{mg}, 5.00 \mathrm{mmol})$ and dioxane $(2 \mathrm{~mL})$ were then added to the reaction mixture. The reaction vessel was placed in the Emrys Optimizer using the following specifications: Temperature: $180{ }^{\circ} \mathrm{C}$, Time: 2700 sec., Fixed Hold Time: On, Sample Absorption: Normal, Pre-stirring: $45 \mathrm{sec}$. After cooling to rt, the reaction mixture was filtered and extracted with $\mathrm{Et}_{2} \mathrm{O}(3 \times 10 \mathrm{~mL})$ and $\mathrm{H}_{2} \mathrm{O}(3 \times 10 \mathrm{~mL})$. The combined organics were washed with brine and dried over anhydrous $\mathrm{MgSO}_{4}$. The solvent was removed in vacuo and the crude product was purified by silica gel chromatography (3:1 hex/EtOAc).

1-Phenylnaphthalene (Table 2, entry 1). Using the standard procedure outlined above, the following amounts of reagents were used: $\mathrm{Ni} / \mathrm{C}_{\mathrm{g}}(48.0 \mathrm{mg}, 0.04 \mathrm{mmol}), \mathrm{PPh}_{3}(31.5 \mathrm{mg}, 0.120 \mathrm{mmol})$, THF (2 $\mathrm{mL}), n$-BuLi $(32 \mu \mathrm{L}, 2.55 \mathrm{M}$ in hexanes, $0.08 \mathrm{mmol}), 2$-tosyloxynaphthalene (149 $\mathrm{mg}, 0.50 \mathrm{mmol})$, phenylboronic acid (122 mg, $1.00 \mathrm{mmol}), \mathrm{KF}$ (87.0 mg, $1.50 \mathrm{mmol}), \mathrm{LiOH}(60.0 \mathrm{mg}, 2.50 \mathrm{mmol})$, and dioxane $(1 \mathrm{~mL})$. Mircowave conditions: Temperature: $200^{\circ} \mathrm{C}$, Time: $7200 \mathrm{sec}$, Fixed Hold Time: On, Sample Absorption: Normal, Pre-stirring: 45 sec. GC-MS analysis indicated $100 \%$ conversion. $^{1}$ 
3,4-Dimethoxy-4'-phenylbiphenyl (Table 2, entry 2). Using the standard procedure outlined above, the following amounts of reagents were used: $\mathrm{Ni} / \mathrm{C}_{\mathrm{g}}(48.0 \mathrm{mg}, 0.04 \mathrm{mmol}), \mathrm{PPh}_{3}(31.5 \mathrm{mg}, 0.120 \mathrm{mmol})$, THF (2 mL), $n$-BuLi $(32 \mu \mathrm{L}, 2.55 \mathrm{M}$ in hexanes, $0.08 \mathrm{mmol})$, biphenyl-4-yl 4-methylbenzenesulfonate (162 mg, $0.50 \mathrm{mmol})$, 3,4-dimethoxyphenylboronic acid (182 mg, $1.00 \mathrm{mmol})$, KF (87.0 mg, 1.50 $\mathrm{mmol}), \mathrm{LiOH}(60.0 \mathrm{mg}, 2.50 \mathrm{mmol})$, and dioxane $(1 \mathrm{~mL})$. Mircowave conditions: Temperature: 180 ${ }^{\circ} \mathrm{C}$, Time: 16200 sec., Fixed Hold Time: On, Sample Absorption: Normal, Pre-stirring: 45 sec. After purification $118 \mathrm{mg}$ of the title compound was isolated $(81 \%) . \mathrm{R}_{\mathrm{f}}=0.29(3: 1 \mathrm{hex} / \mathrm{EtOAc})$. Spectral data on the product matched that of previously reported spectra.

3,4-Dimethoxy-4'-phenylbiphenyl (Table 2, entry 3). Using the standard procedure outlined above, the following amounts of reagents were used: $\mathrm{Ni} / \mathrm{C}_{\mathrm{g}}(48.0 \mathrm{mg}, 0.04 \mathrm{mmol}), \mathrm{PPh}_{3}(31.5 \mathrm{mg}, 0.120 \mathrm{mmol})$, THF (2 mL), $n$-BuLi (32 $\mu \mathrm{L}, 2.55 \mathrm{M}$ in hexanes, $0.08 \mathrm{mmol}$ ), biphenyl-4-yl 4-methylbenzenesulfonate (162 mg, $0.50 \mathrm{mmol}), 3$,4-dimethoxyphenylboronic acid (182 mg, $1.00 \mathrm{mmol}), \mathrm{KF}$ (87.0 mg, 1.50 mmol), LiOH (60.0 mg, $2.50 \mathrm{mmol})$, and dioxane $(1 \mathrm{~mL})$. Mircowave conditions: Temperature: 200 ${ }^{\circ} \mathrm{C}$, Time: 5400 sec., Fixed Hold Time: On, Sample Absorption: Normal, Pre-stirring: 45 sec. After purification on silica gel (3:1 hex:EtOAc), $120 \mathrm{mg}$ of the title compound was isolated $(83 \%) . \mathrm{R}_{\mathrm{f}}=0.29$ (3:1 hex/EtOAc); ${ }^{1} \mathrm{H}$ NMR $\left(\mathrm{CDCl}_{3}, 400 \mathrm{MHz}\right) \delta 7.65(\mathrm{~m}, 6 \mathrm{H}), 7.47(\mathrm{~m}, 2 \mathrm{H}), 7.20(\mathrm{~m}, 1 \mathrm{H}), 7.16(\mathrm{~m}$, $1 \mathrm{H}), 6.99(\mathrm{~s}, 1 \mathrm{H}), 6.96(\mathrm{~s}, 1 \mathrm{H}), 3.98(\mathrm{~s}, 3 \mathrm{H}), 3.94(\mathrm{~s}, 3 \mathrm{H}) ;{ }^{13} \mathrm{C} \mathrm{NMR}\left(\mathrm{CDCl}_{3}, 100 \mathrm{MHz}\right) \delta 149.3,148.8$, 140.1, 139.9, 129.0, 127.6, 127.5, 127.4, 127.2, 119.5, 111.6, 110.4, 56.1; HREIMS $\mathrm{m} / \mathrm{z}$ calcd for $\mathrm{C}_{20} \mathrm{H}_{18} \mathrm{O}_{2} 290.1307$; found 290.1301.

3,4-Dimethoxy-4'-phenylbiphenyl (using S-Phos). Using the standard procedure outlined above, the following amounts of reagents were used: $\mathrm{Ni} / \mathrm{C}_{\mathrm{g}}(48.0 \mathrm{mg}, 0.04 \mathrm{mmol}), 2$-dicyclohexylphosphino-2',6'dimethoxybiphenyl (S-Phos, $15.7 \mathrm{mg}, 0.04 \mathrm{mmol})$, THF (2 mL), $n$-BuLi $(32 \mu \mathrm{L}, 2.55 \mathrm{M}$ in hexanes, $0.08 \mathrm{mmol})$, biphenyl-4-yl 4-methylbenzenesulfonate $(162 \mathrm{mg}, 0.50 \mathrm{mmol})$, 3,4-dimethoxyphenylboronic acid (182 mg, $1.00 \mathrm{mmol}), \mathrm{KF}(87.0 \mathrm{mg}, 1.50 \mathrm{mmol}), \mathrm{LiOH}(60.0 \mathrm{mg}, 2.50 \mathrm{mmol})$, and dioxane ( $1 \mathrm{~mL}$ ). Mircowave conditions: Temperature: $200{ }^{\circ} \mathrm{C}$, Time: $5400 \mathrm{sec}$. , Fixed Hold Time: On, Sample 
Absorption: Normal, Pre-stirring: $45 \mathrm{sec}$. After purification $126 \mathrm{mg}$ of the title compound was isolated $(87 \%) . \mathrm{R}_{\mathrm{f}}=0.29(3: 1 \mathrm{hex} / \mathrm{EtOAc})$. Spectral data on the product matched that of previously reported spectra (listed above).

1-(4'-Benzoylbiphenyl-3-yl)ethanone (Table 2, entry 4). Using the standard procedure outlined above, the following amounts of reagents were used: $\mathrm{Ni} / \mathrm{C}_{\mathrm{g}}(96.0 \mathrm{mg}, 0.08 \mathrm{mmol}), \mathrm{PPh}_{3}(63.0 \mathrm{mg}, 0.24 \mathrm{mmol})$, THF $(2 \mathrm{~mL}), \quad n$-BuLi $(63 \mu \mathrm{L}, \quad 2.55 \mathrm{M}$ in hexanes, $0.16 \mathrm{mmol})$, 4-benzoylphenyl 4methylbenzenesulfonate (352 mg, $1.00 \mathrm{mmol})$, 3-acetylphenylboronic acid (328 mg, $2.00 \mathrm{mmol})$, KF (174 mg, $3.00 \mathrm{mmol})$, LiOH (120 mg, $5.00 \mathrm{mmol})$, and dioxane $(2 \mathrm{~mL})$. Microwave conditions: Temperature: $180{ }^{\circ} \mathrm{C}$, Time: 16200 sec., Fixed Hold Time: On, Sample Absorption: Normal, Prestirring: $45 \mathrm{sec}$. After purification $240 \mathrm{mg}$ of the title compound was isolated $(80 \%)$. $\mathrm{R}_{\mathrm{f}}=0.23(3: 1$ hex/ EtOAc); ${ }^{1} \mathrm{H} \mathrm{NMR}\left(\mathrm{CDCl}_{3}, 400 \mathrm{MHz}\right) \delta 8.25(\mathrm{~s}, 1 \mathrm{H}), 8.01(\mathrm{~m}, 1 \mathrm{H}), 7.93(\mathrm{~m}, 2 \mathrm{H}), 7.85(\mathrm{~m}, 3 \mathrm{H}), 7.75(\mathrm{~m}$, 2H), $7.61(\mathrm{~m}, 2 \mathrm{H}), 7.52(\mathrm{~m}, 2 \mathrm{H}), 2.69(\mathrm{~s}, 3 \mathrm{H}) ;{ }^{13} \mathrm{C} \mathrm{NMR}\left(\mathrm{CDCl}_{3}, 100 \mathrm{MHz}\right) \delta 198.1,196.4,144.3$, $140.7,137.9,137.7,136.9,132.7,132.0,131.0,130.2,129.5,128.5,128.3,127.2,27.0 ;$ HREIMS $m / z$ calcd for $\mathrm{C}_{21} \mathrm{H}_{16} \mathrm{O}_{2} 300.1150$; found 300.1139 .

1-(4'-Methoxybiphenyl-2-yl)ethanone (Table 2, entry 5). Using the standard procedure outlined above, the following amounts of reagents were used: $\mathrm{Ni} / \mathrm{C}_{\mathrm{g}}(48.0 \mathrm{mg}, 0.04 \mathrm{mmol}), \mathrm{PPh}_{3}(31.5 \mathrm{mg}, 0.120$ mmol), THF (2 mL), $n$-BuLi $(32 \mu \mathrm{L}, 2.55 \mathrm{M}$ in hexanes, $0.08 \mathrm{mmol})$, 2-acetylphenyl-4methylbenzenesulfonate (145 mg, $0.50 \mathrm{mmol})$, 4-methoxyphenylboronic acid (152 mg, $1.00 \mathrm{mmol}), \mathrm{KF}$ (87 mg, $1.50 \mathrm{mmol}), \mathrm{LiOH}(60 \mathrm{mg}, 2.50 \mathrm{mmol})$, and dioxane $(1 \mathrm{~mL})$. Microwave conditions: Temperature: $200^{\circ} \mathrm{C}$, Time: 5400 sec., Fixed Hold Time: On, Sample Absorption: Normal, Pre-stirring: $45 \mathrm{sec}$. After purification $90 \mathrm{mg}$ of the title compound was isolated $(80 \%) . \mathrm{R}_{\mathrm{f}}=0.40(3: 1 \mathrm{hex} / \mathrm{EtOAc})$; ${ }^{1} \mathrm{H} \mathrm{NMR}\left(\mathrm{CDCl}_{3}, 400 \mathrm{MHz}\right) \delta 7.51(\mathrm{~m}, 2 \mathrm{H}), 7.386(\mathrm{~m}, 2 \mathrm{H}), 7.26(\mathrm{~m}, 2 \mathrm{H}), 6.98(\mathrm{~m}, 2 \mathrm{H}), 3.86(\mathrm{~s}, 3 \mathrm{H})$, $2.02(\mathrm{~s}, 3 \mathrm{H}) ;{ }^{13} \mathrm{C} \mathrm{NMR}\left(\mathrm{CDCl}_{3}, 100 \mathrm{MHz}\right) \delta 205.6,159.7,141.1,140.3,133.2,130.9,130.3,130.2$ 128.0, 127.2, 114.3, 55.5, 30.6; HREIMS m/z calcd for $\mathrm{C}_{15} \mathrm{H}_{14} \mathrm{O}_{2} 226.0994$; found 226.0998. 
1-(3'-(Trifluoromethyl)biphenyl-2-yl)ethanone (Table 2, entry 6). Using the standard procedure outlined above, the following amounts of reagents were used: $\mathrm{Ni} / \mathrm{C}_{\mathrm{g}}(48.0 \mathrm{mg}, 0.04 \mathrm{mmol}), \mathrm{PPh}_{3}(31.5$ mg, $0.120 \mathrm{mmol})$, THF (2 mL), $n$-BuLi $(32 \mu \mathrm{L}, 2.55 \mathrm{M}$ in hexanes, $0.08 \mathrm{mmol})$, 2-acetylphenyl-4methylbenzenesulfonate (145 mg, $0.50 \mathrm{mmol})$, 3-(trifluoromethyl)phenylboronic acid (190 mg, 1.00 $\mathrm{mmol}), \mathrm{KF}$ (87 mg, $1.50 \mathrm{mmol}), \mathrm{LiOH}(60 \mathrm{mg}, 2.50 \mathrm{mmol})$, and dioxane (1 mL). Microwave conditions: Temperature: $200^{\circ} \mathrm{C}$, Time: $5400 \mathrm{sec}$. Fixed Hold Time: On, Sample Absorption: Normal, Pre-stirring: $45 \mathrm{sec}$. After purification $120 \mathrm{mg}$ of the title compound was isolated $(91 \%) . \mathrm{R}_{\mathrm{f}}=0.33(3: 1$ hex/EtOAc); ${ }^{1} \mathrm{H}$ NMR $\left(\mathrm{CDCl}_{3}, 400 \mathrm{MHz}\right) \delta 7.64(\mathrm{~m}, 3 \mathrm{H}), 7.55(\mathrm{~m}, 2 \mathrm{H}), 7.49(\mathrm{~m}, 2 \mathrm{H}), 7.38(\mathrm{~m}, 1 \mathrm{H})$, $2.18(\mathrm{~s}, 3 \mathrm{H}) ;{ }^{13} \mathrm{C} \mathrm{NMR}\left(\mathrm{CDCl}_{3}, 100 \mathrm{MHz}\right) \delta 203.7,141.8,139.3,132.4,131.3,130.7,129.2,128.5$, 128.3, 125.6, 124.7, 30.5; HREIMS $m / z$ calcd for $\mathrm{C}_{15} \mathrm{H}_{11} \mathrm{~F}_{3} \mathrm{O} 264.0762$; found 264.0771.

\section{Negishi Carboalumination of a terminal alkyne using catalytic $\mathrm{Cp}_{2} \mathrm{ZrCl}_{2}$ / catalytic IBAO / excess}

\section{$\mathrm{Me}_{3} \mathrm{Al}$ followed by $\mathrm{Ni} / \mathrm{C}_{\mathrm{g}}$ catalyzed cross-coupling with an aryl tosylate; $(E)$-4-(2-methyloct-1-} enyl)biphenyl (Scheme 3). To a flame dried argon purged $25 \mathrm{~mL}$ round bottomed (rb) flask was added bis(cyclopentadienyl)zirconium dichloride $(14.6 \mathrm{mg}, 0.05 \mathrm{mmol}, 5.0 \mathrm{~mol} \%)$, followed by the dropwise addition at $0{ }^{\circ} \mathrm{C}$ of $\mathrm{Me}_{3} \mathrm{Al}(2.0 \mathrm{M}$ solution in toluene, $0.75 \mathrm{~mL}, 1.50 \mathrm{mmol}, 1.5$ equiv). While stirring at $0{ }^{\circ} \mathrm{C}$, IBAO (0.28 mL, $\left.0.100 \mathrm{mmol}, 10 \mathrm{~mol} \%\right)$ was then added. Lastly, octyne $(0.150 \mathrm{~mL}, 1.00 \mathrm{mmol})$ was introduced and the homogeneous pale yellow solution stirred at $\mathrm{rt}$ for $2 \mathrm{~h}$ until TLC analysis (5\% $\mathrm{CH}_{2} \mathrm{Cl}_{2}$ /pet ether) indicated that the carboalumination was complete.

In a separate flame dried argon purged $10 \mathrm{~mL}$ microwave vial was combined triphenylphosphine (32 $\mathrm{mg}, 0.12 \mathrm{mmol})$ and $\mathrm{Ni} / \mathrm{C}_{\mathrm{g}}(48 \mathrm{mg}, 0.03 \mathrm{mmol}, 0.03$ equiv) in the glove box. THF $(0.5 \mathrm{~mL})$ was then added via syringe followed by the dropwise addition of $n$-BuLi ( $20.5 \mu \mathrm{L}, 0.06 \mathrm{mmol})$ to form the active $\mathrm{Ni}(0) / \mathrm{C}_{\mathrm{g}}$ complex. After stirring at $\mathrm{rt}$ for $5 \mathrm{~min}$, the vinylalane generated above was transferred to the vial via cannula. Lastly, 4-tosyloxybiphenyl (162.2 $\mathrm{mg}, 0.5 \mathrm{mmol})$ was added and the reaction vessel was placed in the Emry's Optimizer and exposed to microwave irradiation according to the following specifications: Temperature: $200{ }^{\circ} \mathrm{C}$, Time: 3600 sec., Fixed Hold Time: On, Sample Absorption: 
Normal, Pre-stirring: $45 \mathrm{sec}$. The reaction mixture was then extracted with water $(3 \times 10 \mathrm{~mL})$ and $\mathrm{Et}_{2} \mathrm{O}$ ( $3 \times 10 \mathrm{~mL}$ ). The combined organics were washed with brine and dried over anhydrous $\mathrm{MgSO}_{4}$. The solvent was removed in vacuo and the crude product was purified by silica gel chromatography (3:1 hex/ EtOAc) yielding $116 \mathrm{mg}$ of compound $\mathbf{1 0}$ as a colorless oil (83\%). The other regioisomer was not detected by GC. $\mathrm{R}_{\mathrm{f}}=0.70\left(3: 1\right.$ hex/EtOAc); ${ }^{1} \mathrm{H}$ NMR $\left(\mathrm{CDCl}_{3}, 400 \mathrm{MHz}\right) \delta 7.50(\mathrm{~m}, 2 \mathrm{H}), 7.46(\mathrm{~m}, 2 \mathrm{H})$, $7.35(\mathrm{~m}, 4 \mathrm{H}), 7.20(\mathrm{~s}, 1 \mathrm{H}), 6.17(\mathrm{~m}, 1 \mathrm{H}), 1.92(\mathrm{~m}, 2 \mathrm{H}), 1.70(\mathrm{~s}, 3 \mathrm{H}), 1.30(\mathrm{~m}, 5 \mathrm{H}), 1.23(\mathrm{~m}, 3 \mathrm{H}), 0.91$ (m, 3H); ${ }^{13} \mathrm{C} \mathrm{NMR}\left(\mathrm{CDCl}_{3}, 100 \mathrm{MHz}\right) \delta 137.3,136.0,134.2,131.1,129.8,128.6,127.5$ 127.4, 123.6, 115.3, 33.2, 31.9, 29.5, 29.1, 22.8, 15.8, 14.3; HREIMS $m / z$ calcd for $\mathrm{C}_{21} \mathrm{H}_{26}$ 278.2034; found 278.2036. Standard Ni/ $\mathrm{C}_{\mathrm{g}}$ catalyzed cross-couplings of vinylzirconocenes with aryl halides. To a flame dried, argon purged $25 \mathrm{~mL}$ round bottom (rb) flask were added bis(cyclopentadienyl)zirconium chloride hydride ( $135 \mathrm{mg}, 0.50 \mathrm{mmol})$ in the glove box, followed by THF $(1.0 \mathrm{~mL})$ via syringe. Octyne (0.075 $\mathrm{mL}, 0.50 \mathrm{mmol}$ ) was then added and the reaction was allowed to stir at $\mathrm{rt}$ until $\mathrm{GC}$ analysis indicated complete disappearance of the starting material. In a separate flame dried argon purged $10 \mathrm{~mL}$ microwave vial was combined triphenylphosphine $(21 \mathrm{mg}, 0.08 \mathrm{mmol})$ and $\mathrm{Ni} / \mathrm{C}_{\mathrm{g}}(48 \mathrm{mg}, 0.04 \mathrm{mmol})$ in the glove box. THF $(0.5 \mathrm{~mL})$ was then added via syringe followed by the dropwise addition of $n$-BuLi (30.75 $\mu \mathrm{L}, 0.08 \mathrm{mmol})$ to form the active $\mathrm{Ni}(0) / \mathrm{C}_{\mathrm{g}}$ complex. After stirring at $\mathrm{rt}$ for $5 \mathrm{~min}$, the vinylzirconocene generated above was transferred to the vial via cannula. Lastly, the aryl halide $(0.50$ mmol) was added and the reaction vessel was placed in the Emry's Optimizer and exposed to microwave irradiation according to the following specifications: Temperature: $200{ }^{\circ} \mathrm{C}$, Time: $900 \mathrm{sec}$., Fixed Hold Time: On, Sample Absorption: Normal, Pre-stirring: 45 sec.

(E)-1-(Oct-1-enyl)-3-(trifluoromethyl)benzene (Table 3, entry 1). Using the standard procedure outlined above, the following amounts of reagents were used: zirconocene (135 mg, $0.50 \mathrm{mmol})$, octyne (0.075 mL, $0.5 \mathrm{mmol}), \mathrm{Ni} / \mathrm{C}_{\mathrm{g}}(48 \mathrm{mg}, 0.04 \mathrm{mmol}, 0.1$ equiv), $n$-BuLi (30.75 $\mu \mathrm{L}, 0.08 \mathrm{mmol}, 0.2$ equiv), and 1-iodo-3-(triflouromethyl)benzene $(0.072 \mathrm{~mL}, 0.50 \mathrm{mmol})$. The hydrozirconation was allowed to proceed for 45 min and the reaction vessel was then placed in the Emrys Optimizer using the following 
specifications: Temperature: $200{ }^{\circ} \mathrm{C}$, Time: 600 sec., Fixed Hold Time: On, Sample Absorption: Normal, Pre-stirring: $45 \mathrm{sec}$. The reaction mixture was then extracted with water $(3 \mathrm{x} 10 \mathrm{~mL})$ and $\mathrm{Et}_{2} \mathrm{O}$ (3 x $10 \mathrm{~mL}$ ). The combined organics were washed with brine and dried over anhydrous $\mathrm{MgSO}_{4}$. The solvent was removed in vacuo and the crude product was purified by silica gel chromatography (hexanes) affording $110 \mathrm{mg}$ of the title compound as a colorless oil $(85 \%) . \mathrm{R}_{\mathrm{f}}=0.60$ (hexanes). ${ }^{1} \mathrm{H}$ NMR $\left(\mathrm{CDCl}_{3}, 400 \mathrm{MHz}\right) \delta 7.60(\mathrm{~s}, 1 \mathrm{H}), 7.51(\mathrm{~m}, 1 \mathrm{H}), 7.41(\mathrm{~m}, 1 \mathrm{H}), 7.29(\mathrm{~m}, 1 \mathrm{H}), 6.40(\mathrm{~m}, 1 \mathrm{H}), 6.25(\mathrm{~m}, 1 \mathrm{H})$, $2.21(\mathrm{q}, J=6.0 \mathrm{~Hz}, 2 \mathrm{H}), 1.43(\mathrm{~m}, 2 \mathrm{H}), 1.30(\mathrm{~m}, 6 \mathrm{H}), 0.91(\mathrm{~m}, 3 \mathrm{H}) ;{ }^{13} \mathrm{C} \mathrm{NMR}\left(\mathrm{CDCl}_{3}, 100 \mathrm{MHz}\right) \delta 141.1$, $138.8,133.5,129.2,129.0,128.6,123.9,122.7,33.2,31.9,31.8,29.3,29.1,22.9,14.3 ;$ HREIMS m/z calcd for $\mathrm{C}_{15} \mathrm{H}_{19} \mathrm{~F}_{3} 256.1439$; found 256.1442.

(E)-1-Fluoro-4-(oct-1-enyl)benzene (Table 3, entry 2). Using the standard procedure outlined above, the following amounts of reagents were used: zirconocene $(135 \mathrm{mg}, 0.50 \mathrm{mmol})$, octyne $(0.075 \mathrm{~mL}, 0.5$ mmol), Ni/C $0.08 \mathrm{mmol}, 0.2$ equiv), and 1-bromo-4-fluorobenzene $(0.044 \mathrm{~mL}, 0.40 \mathrm{mmol})$. The hydrozirconation was allowed to proceed for $45 \mathrm{~min}$ and the reaction was then subjected to microwave irradiation according to the following specifications: Temperature: $200{ }^{\circ} \mathrm{C}$, Time: $900 \mathrm{sec}$., Fixed Hold Time: On, Sample Absorption: Normal, Pre-stirring: $45 \mathrm{sec}$. The reaction mixture was then extracted with water (3 x $10 \mathrm{~mL})$ and $\mathrm{Et}_{2} \mathrm{O}(3 \times 10 \mathrm{~mL})$. The combined organics were washed with brine and dried over anhydrous $\mathrm{MgSO}_{4}$. The solvent was removed in vacuo and the crude product was purified by silica gel chromatography (3:1 hex/EtOAc), isolating $171 \mathrm{mg}$ of the title compound as a colorless oil $(83 \%)$. $\mathrm{R}_{\mathrm{f}}=$ 0.67 (3:1 hex/EtOAc); ${ }^{1} \mathrm{H}$ NMR $\left(\mathrm{CDCl}_{3}, 400 \mathrm{MHz}\right) \delta 7.35(\mathrm{~m}, 2 \mathrm{H}), 6.95(\mathrm{~m}, 2 \mathrm{H}), 6.38(\mathrm{~m}, 1 \mathrm{H}), 6.17$ $(\mathrm{m}, 1 \mathrm{H}), 2.18(\mathrm{q}, J=6.1 \mathrm{~Hz}, 2 \mathrm{H}), 1.23(\mathrm{~m}, 8 \mathrm{H}), 0.91(\mathrm{~m}, 3 \mathrm{H}) ;{ }^{13} \mathrm{C} \mathrm{NMR}\left(\mathrm{CDCl}_{3}, 100 \mathrm{MHz}\right) \delta 163.2$ 134.2, 131.1, 128.6, 127.5 127.4, 115.5, 115.3, 33.2, 31.9, 29.5, 29.1, 22.8, 14.3; HREIMS $\mathrm{m} / \mathrm{z}$ calcd for $\mathrm{C}_{14} \mathrm{H}_{19} \mathrm{~F}$ 206.1471; found 206.1475. 
(E)-1-(Oct-1-enyl)-3-(trifluoromethyl)benzene (Table 3, entry 3). Using the standard procedure outlined above, the following amounts of reagents were used: zirconocene (135 mg, $0.50 \mathrm{mmol})$, octyne (0.075 mL, $0.5 \mathrm{mmol}), \mathrm{Ni} / \mathrm{C}_{\mathrm{g}}$ (48 mg, $0.04 \mathrm{mmol}, 0.1$ equiv), $n$-BuLi (30.75 $\mu \mathrm{L}, 0.08 \mathrm{mmol}, 0.2$ equiv), and 1-chloro-3-(trifluoromethyl)benzene $(0.068 \mathrm{~mL}, 0.50 \mathrm{mmol})$. The hydrozirconation was allowed to proceed for $45 \mathrm{~min}$ and the reaction vessel was then placed in the Emrys Optimizer using the following specifications: Temperature: $200{ }^{\circ} \mathrm{C}$, Time: 600 sec., Fixed Hold Time: On, Sample Absorption: Normal, Pre-stirring: $45 \mathrm{sec}$. The reaction mixture was then extracted with water $(3 \mathrm{x} 10 \mathrm{~mL})$ and $\mathrm{Et}_{2} \mathrm{O}$ (3 x $10 \mathrm{~mL}$ ). The combined organics were washed with brine and dried over anhydrous $\mathrm{MgSO}_{4}$. The solvent was removed in vacuo and the crude product was purified by silica gel chromatography (hexanes), affording $115 \mathrm{mg}$ of the title compound as a colorless oil $(90 \%) . \mathrm{R}_{\mathrm{f}}=0.60$ (hexanes). ${ }^{1} \mathrm{H}$ $\operatorname{NMR}\left(\mathrm{CDCl}_{3}, 400 \mathrm{MHz}\right) \delta 7.58(\mathrm{~m}, 1 \mathrm{H}), 7.51(\mathrm{~m}, 1 \mathrm{H}), 7.41(\mathrm{~m}, 2 \mathrm{H}), 6.40(\mathrm{~m}, 1 \mathrm{H}), 6.35(\mathrm{~m}, 1 \mathrm{H}), 2.21$ $(\mathrm{m}, 2 \mathrm{H}), 1.47(\mathrm{~m}, 2 \mathrm{H}), 1.30(\mathrm{~m}, 8 \mathrm{H}), 0.89(\mathrm{~m}, 3 \mathrm{H}) ;{ }^{13} \mathrm{C} \mathrm{NMR}\left(\mathrm{CDCl}_{3}, 100 \mathrm{MHz}\right) \delta 138.8,133.5,129.2$ 129.0, 128.6, 122.7, 33.2, 31.9, 31.8, 29.3, 29.1, 22.8, 14.3; HREIMS $m / z$ calcd for $\mathrm{C}_{15} \mathrm{H}_{19} \mathrm{~F}_{3}$ 256.1439; found 256.1442 .

${ }^{1 .}$ Riggleman, S.; Deshong, P.; J. Org. Chem. 2003, 68, 8106. 


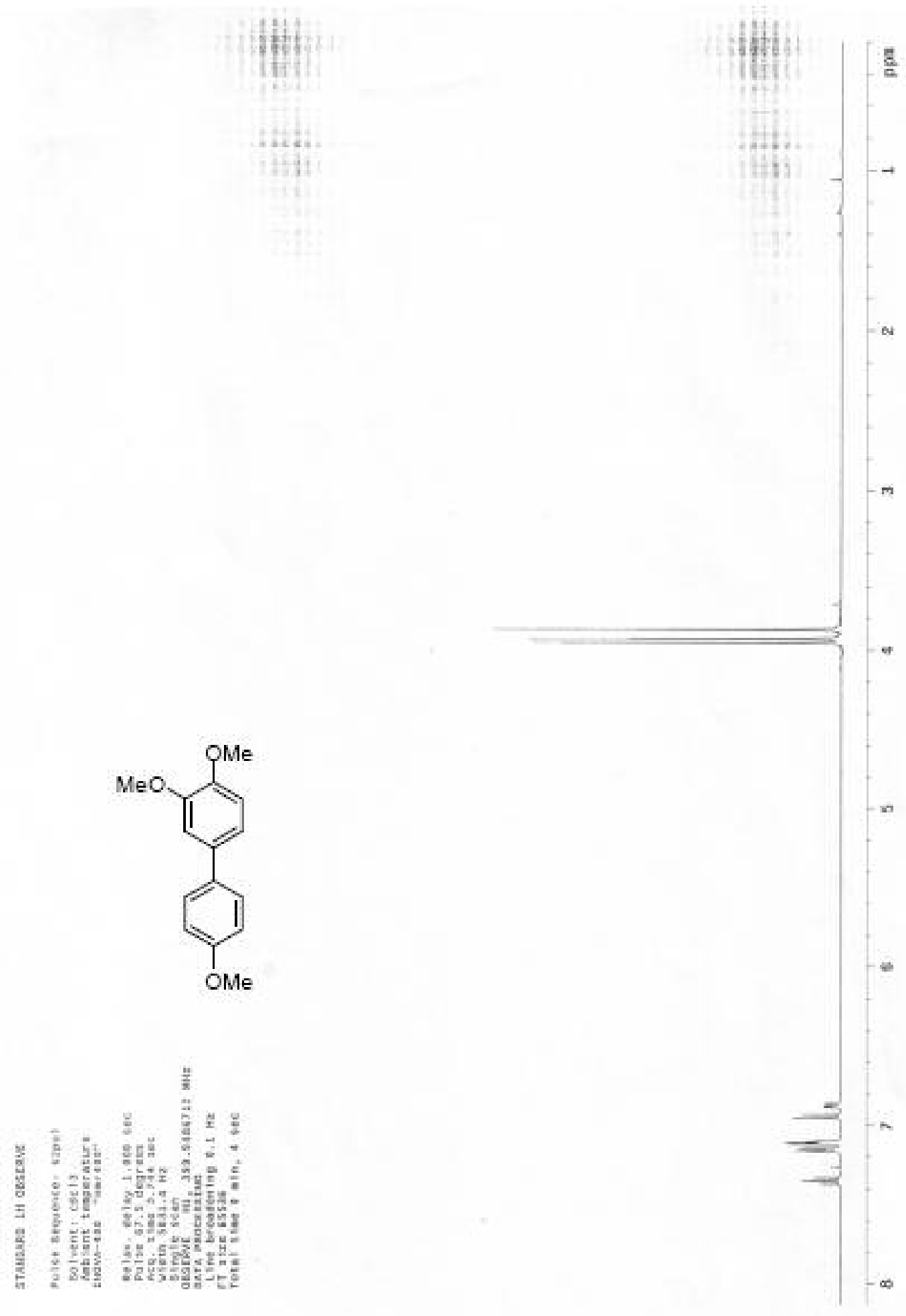

S-13 

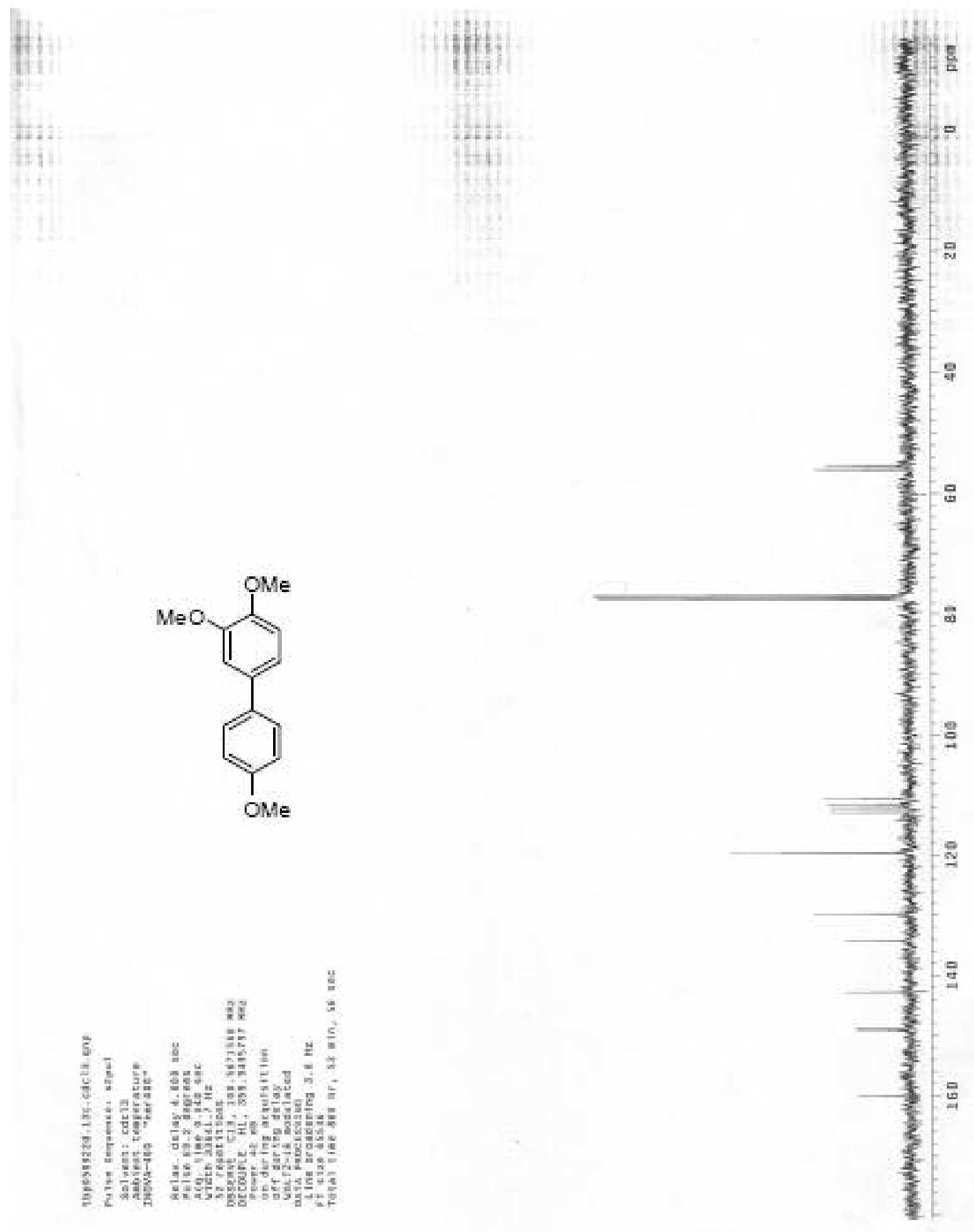


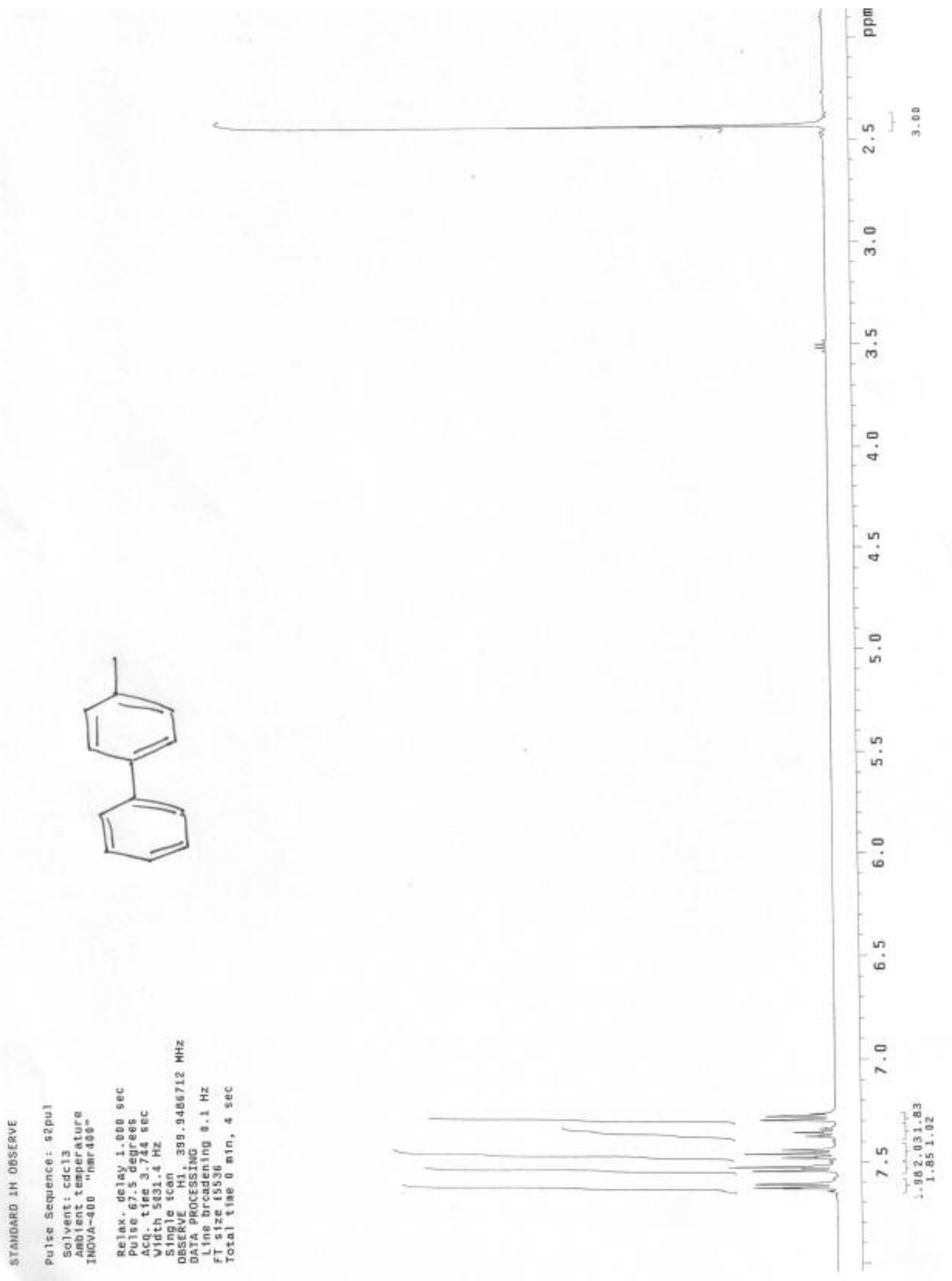

S-15 


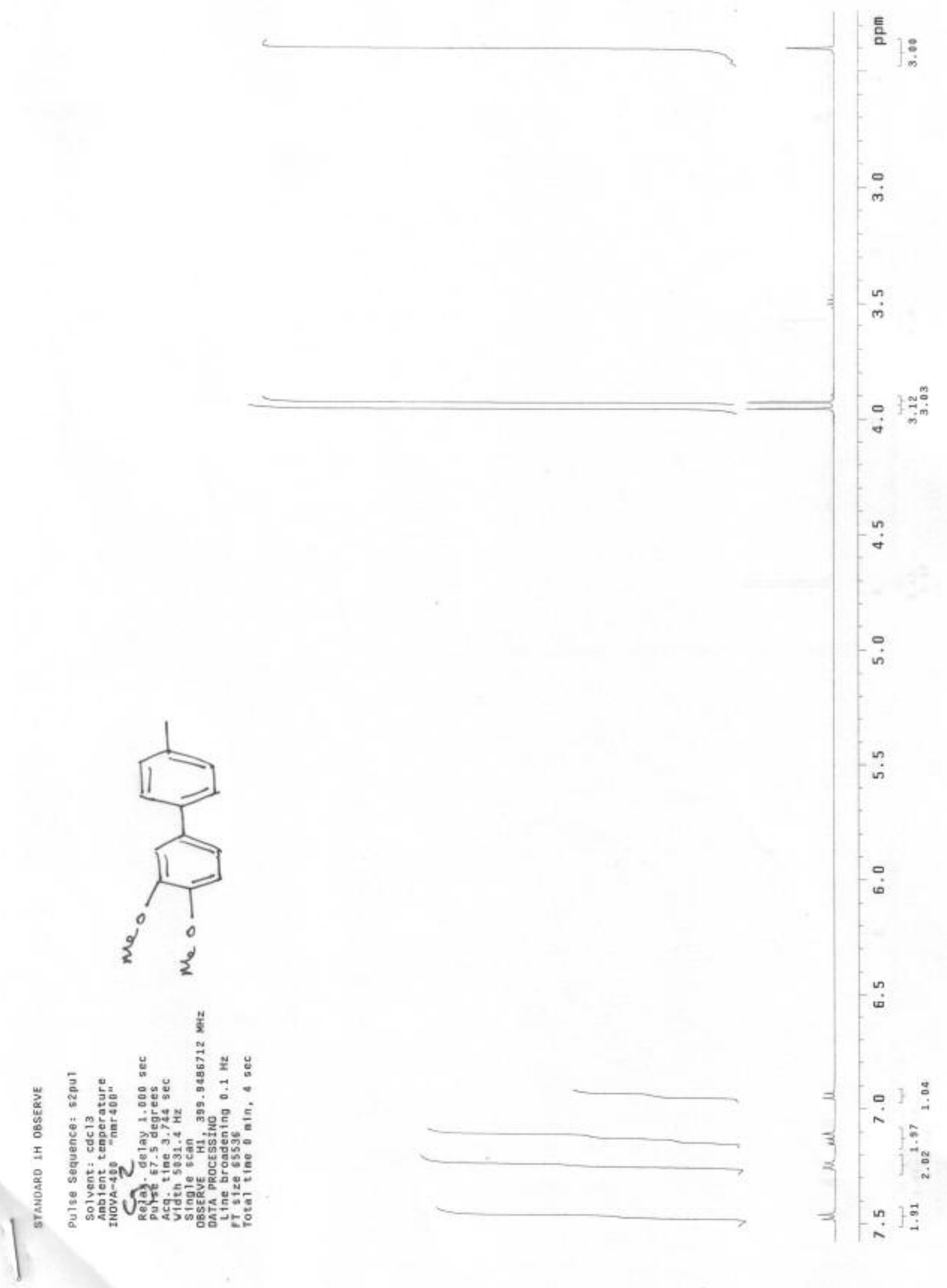

S-16 
<smiles>COc1ccc(-c2ccc(C)cc2)cc1O</smiles>

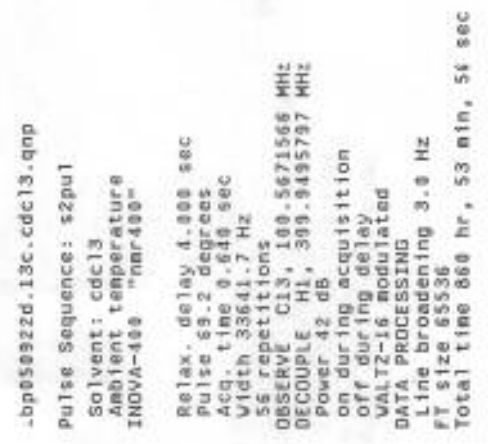

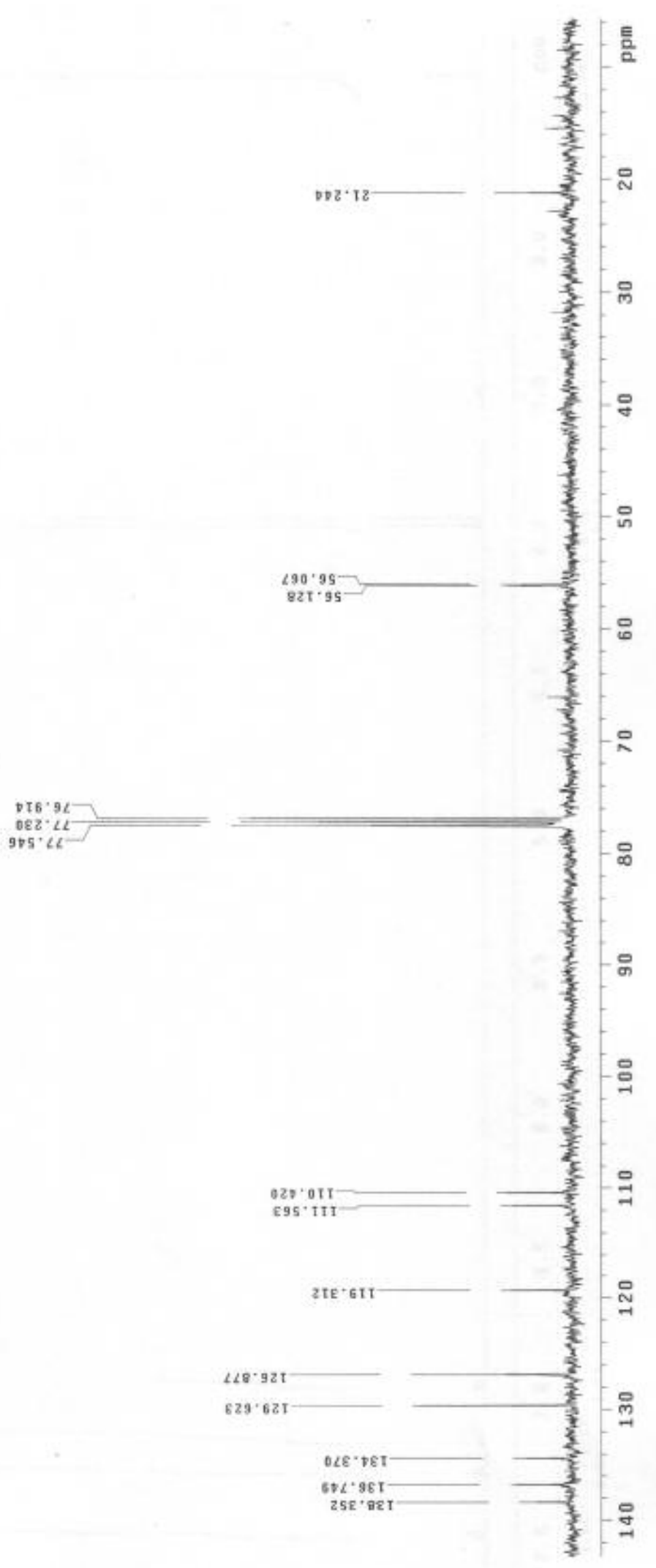




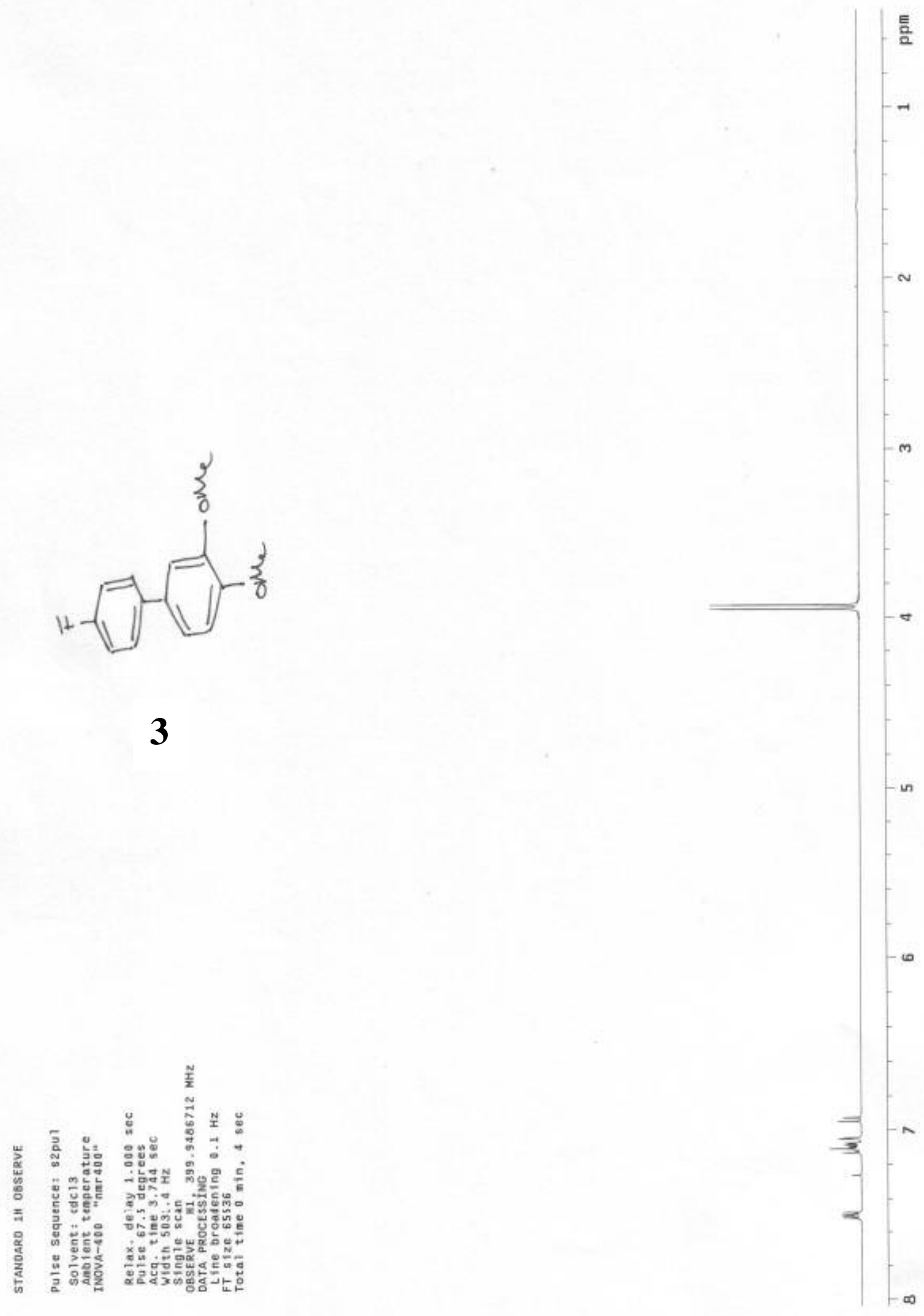

S-18 


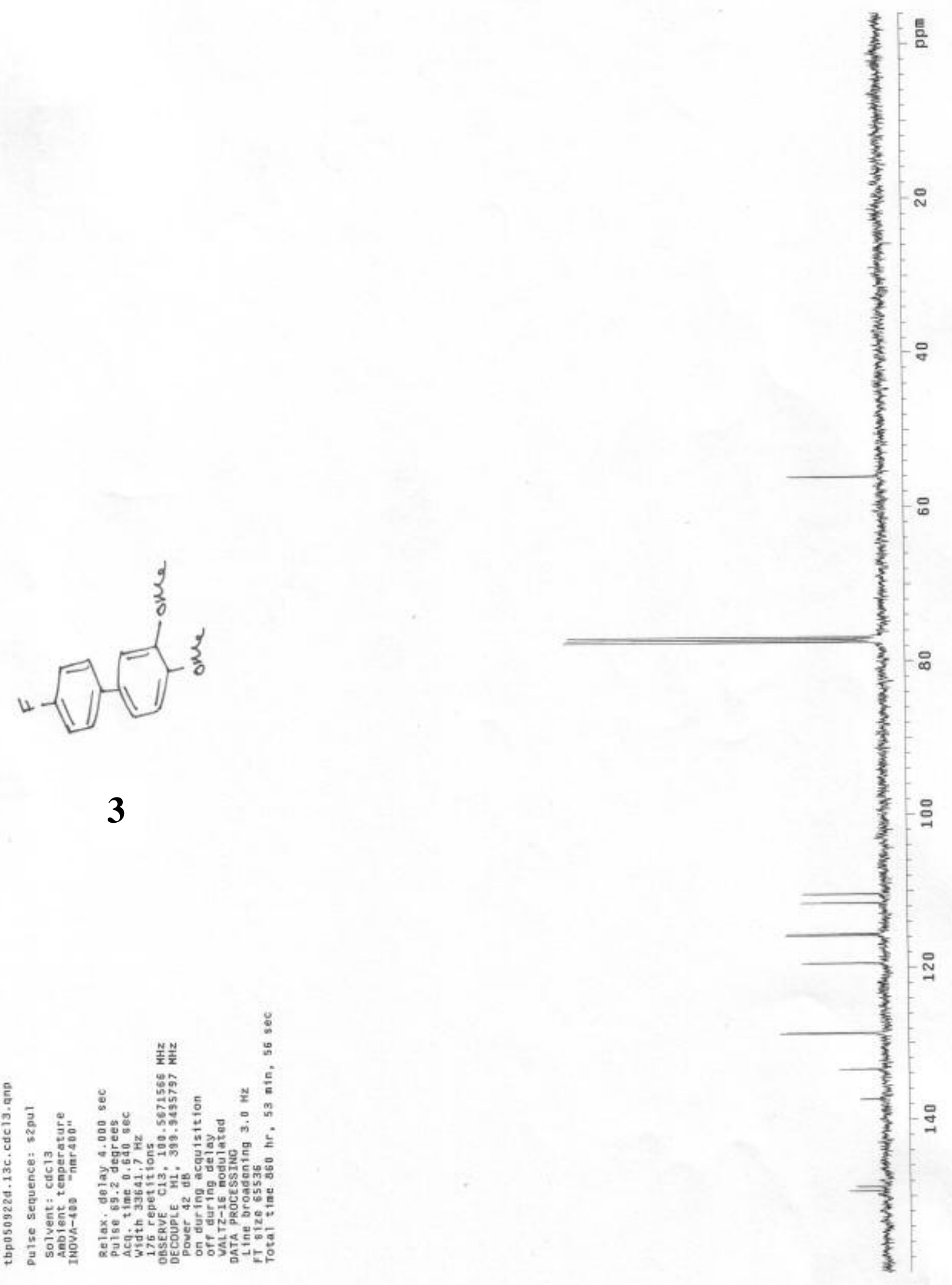

S-19 

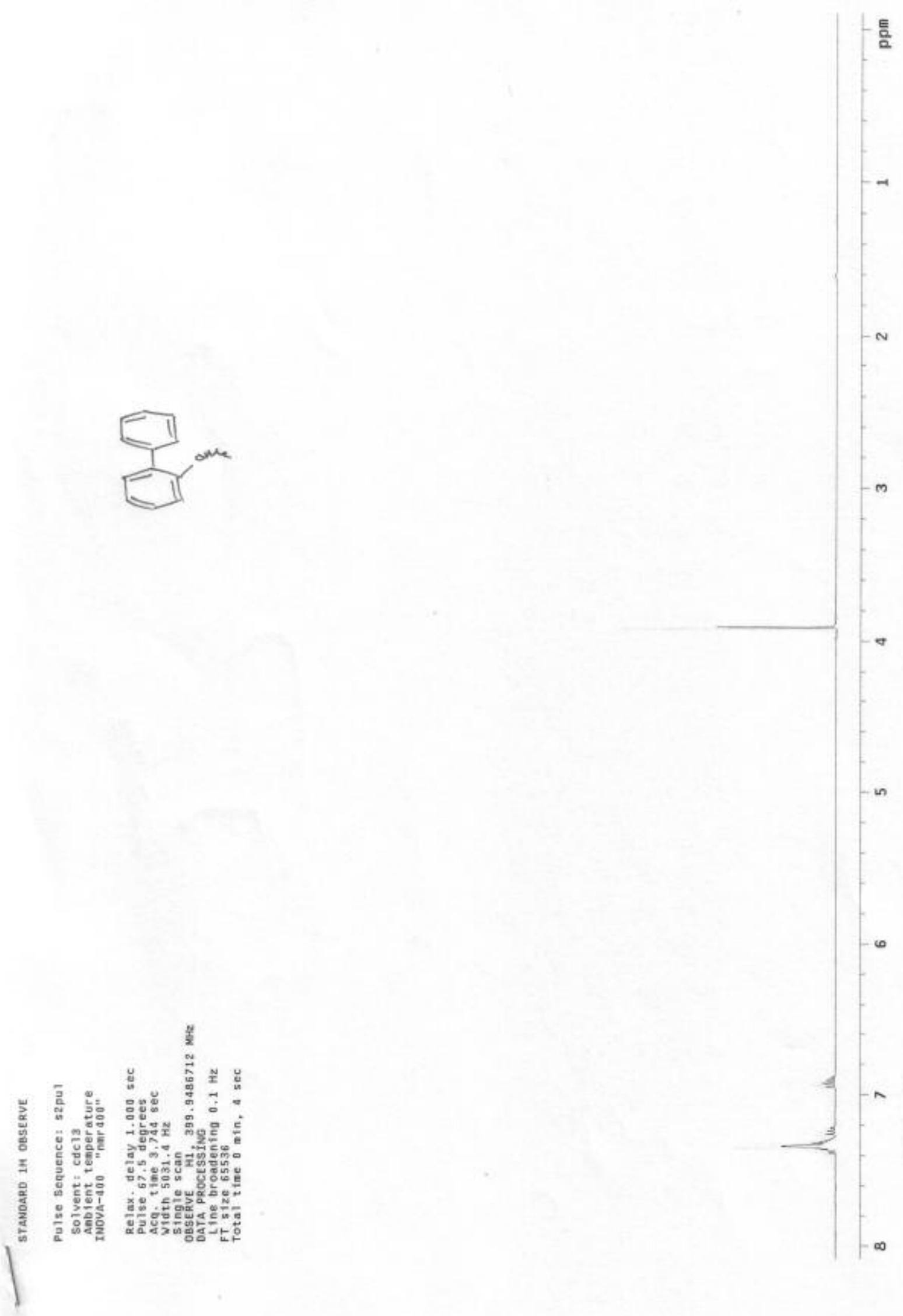

S-20 

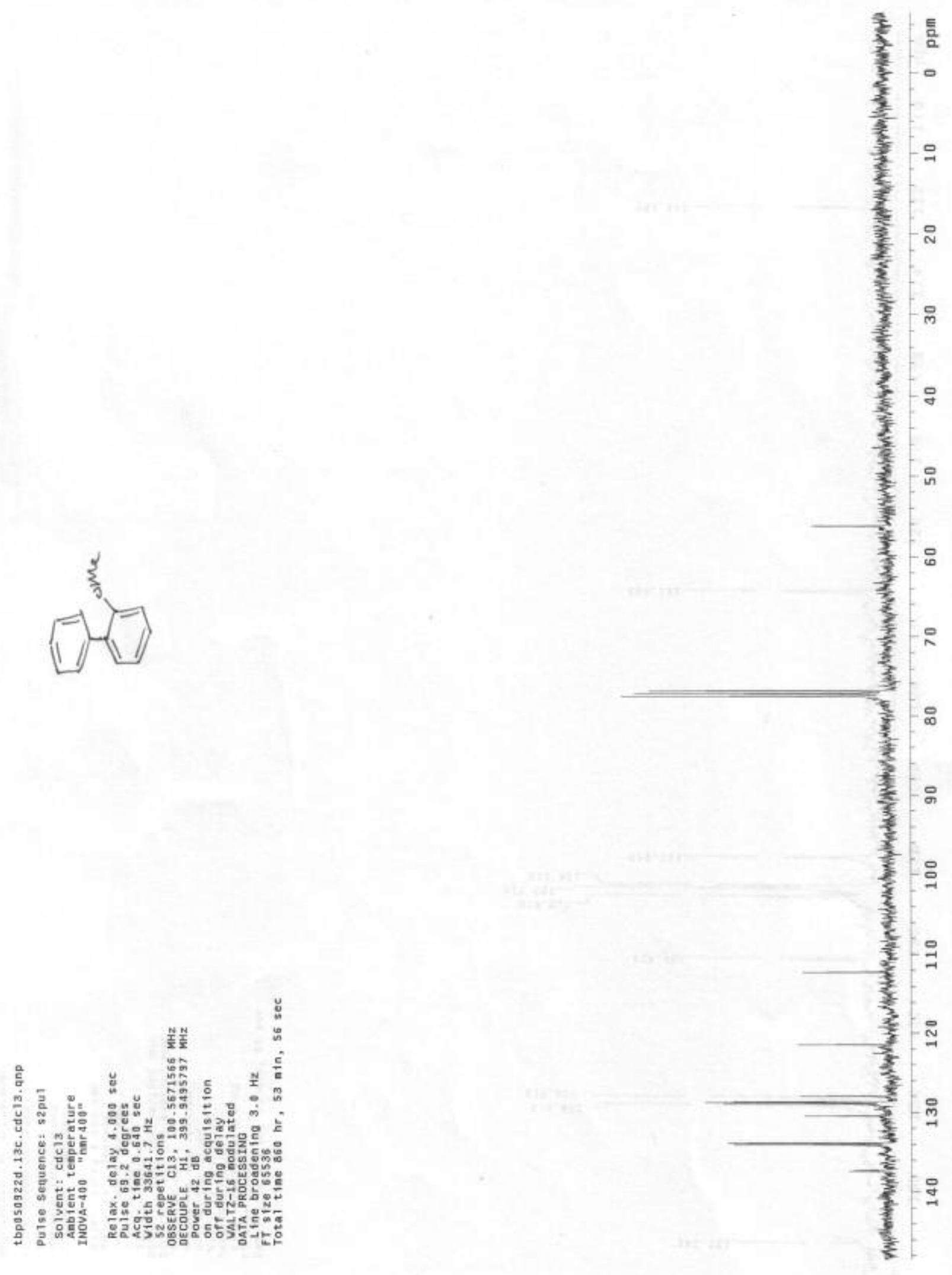

I 

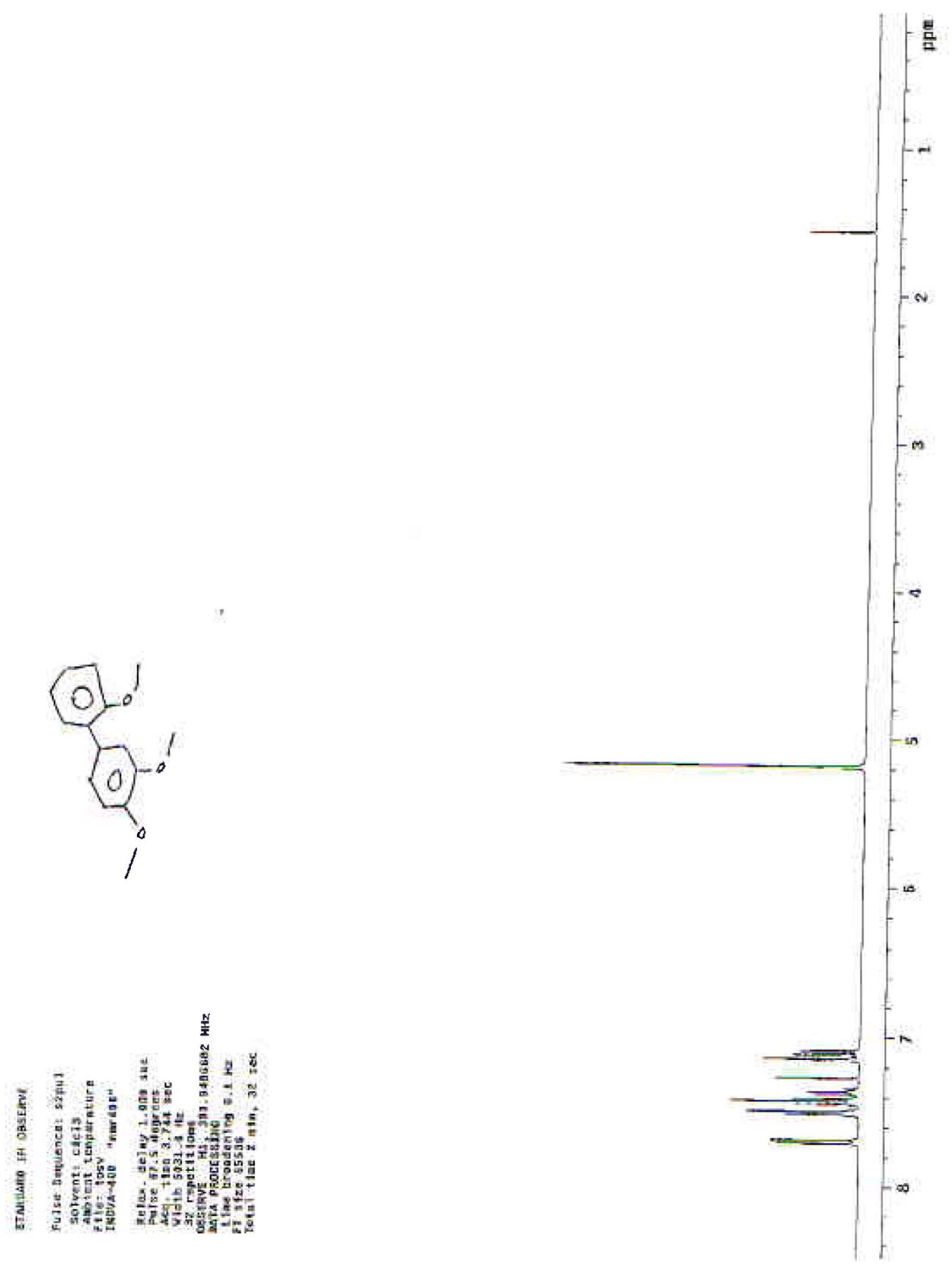


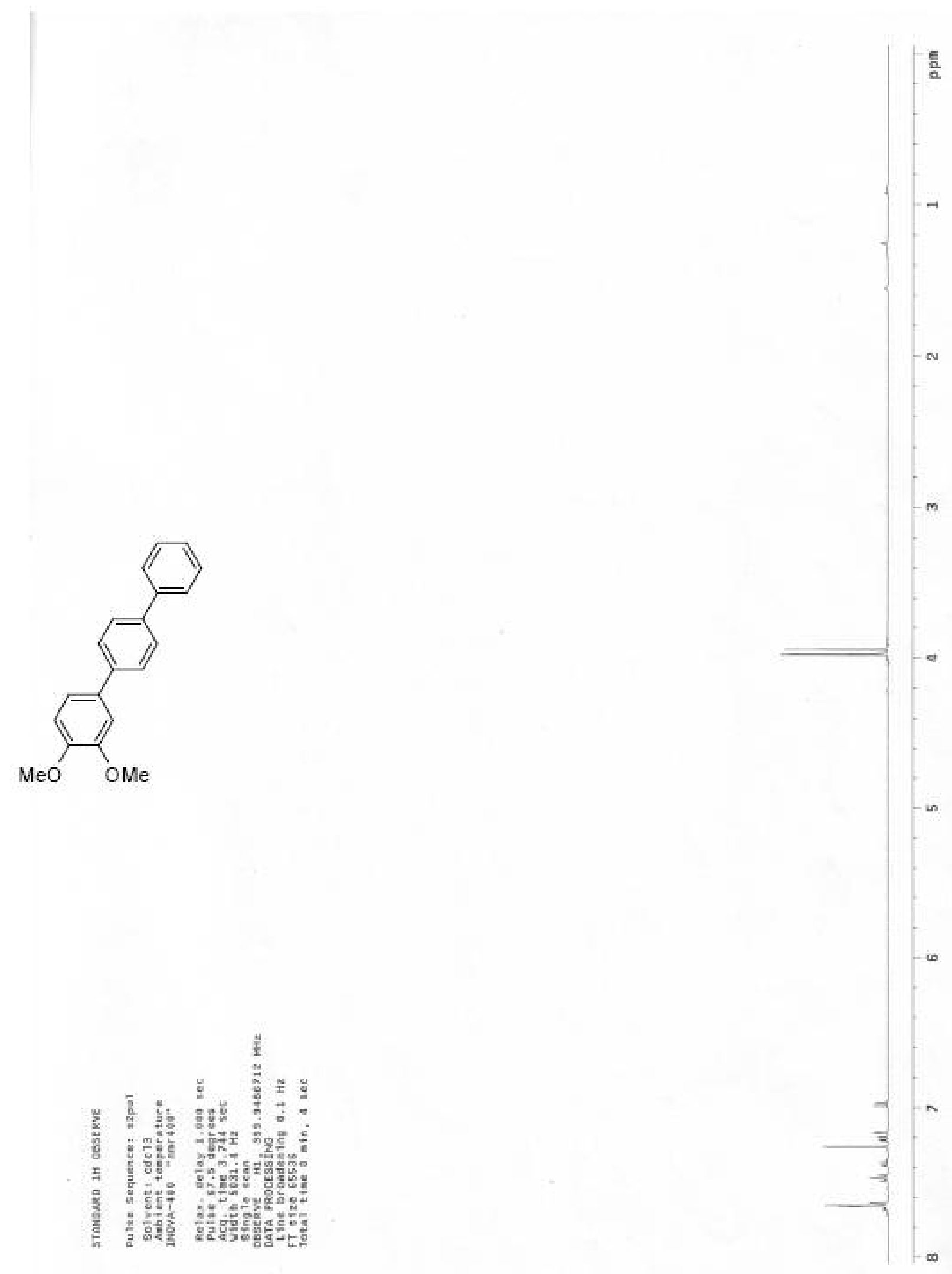

S-23 


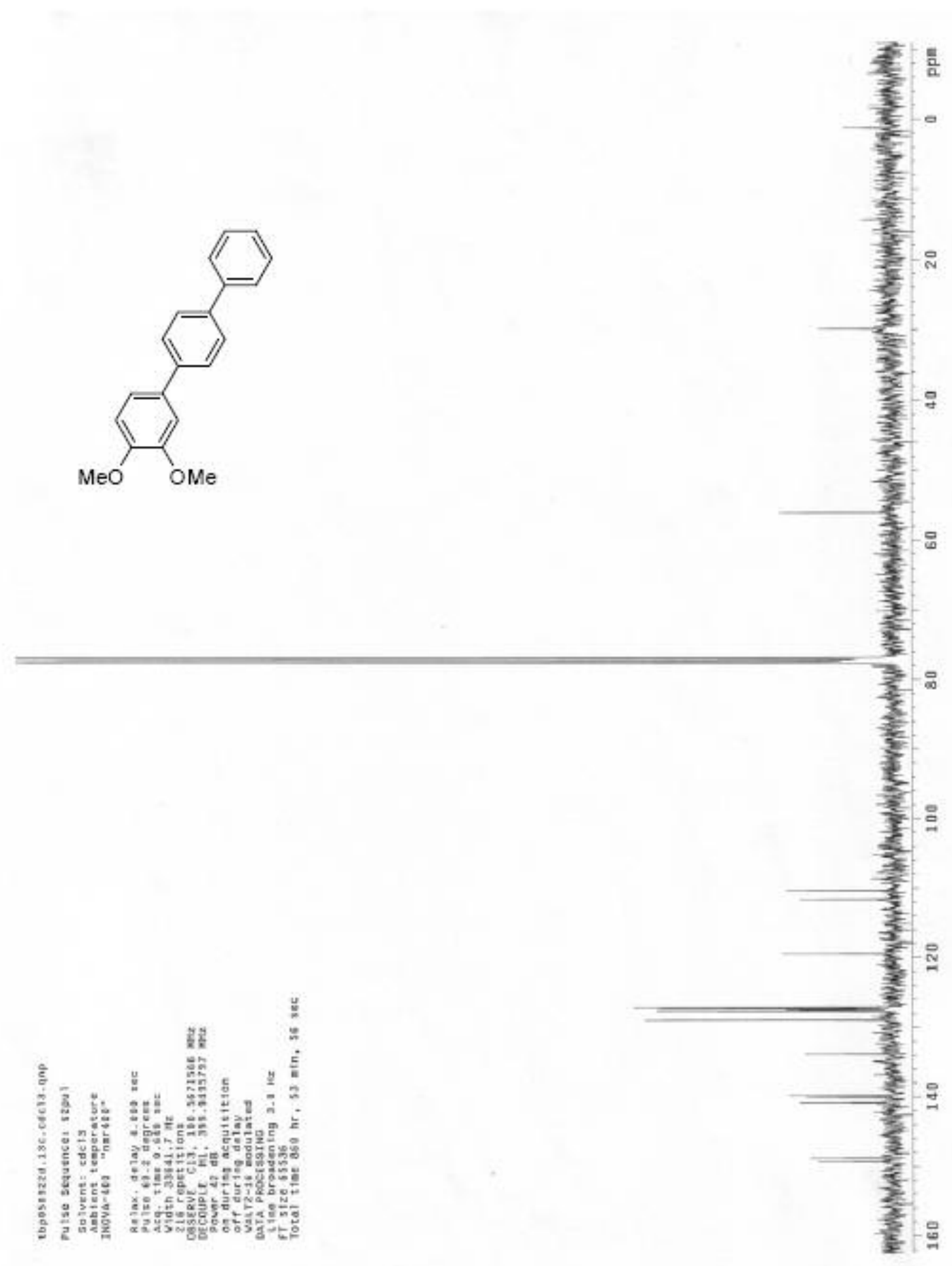




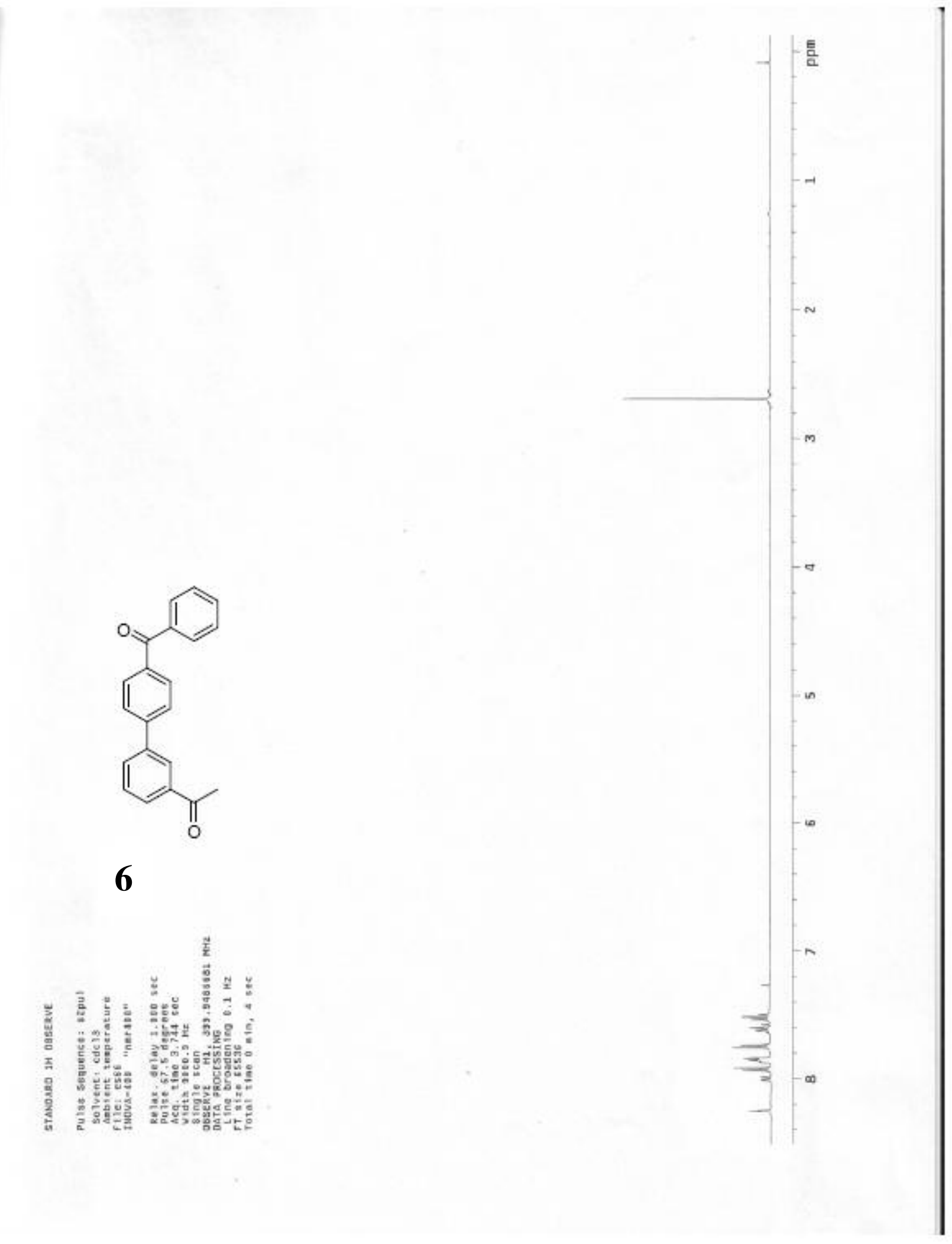

S-25 


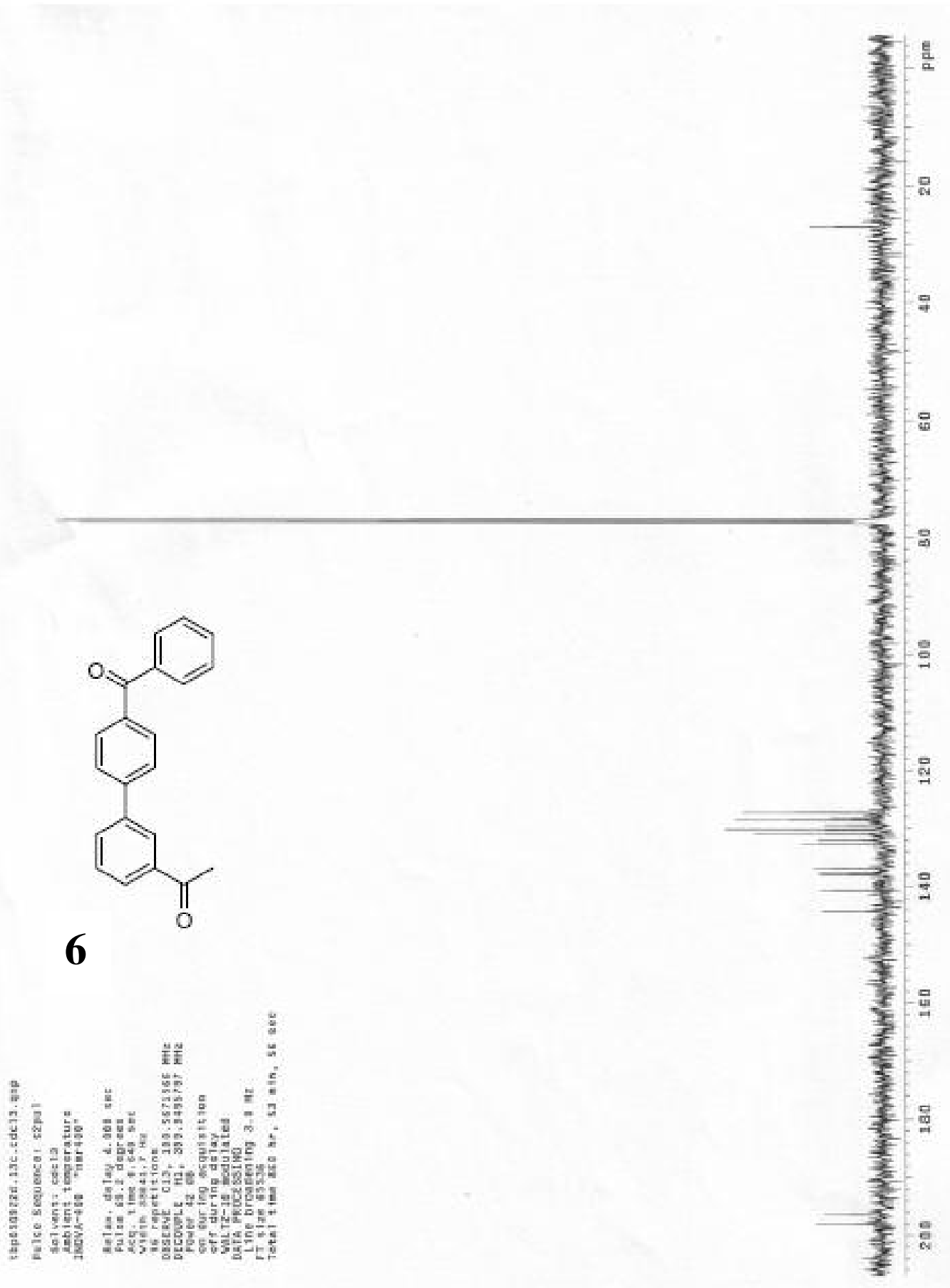

S-26 


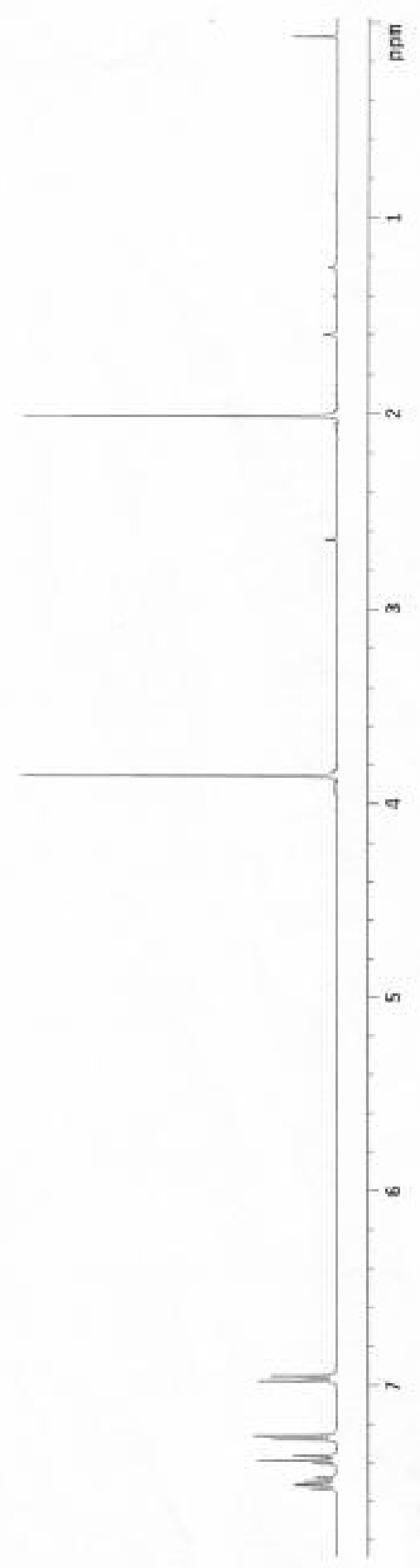




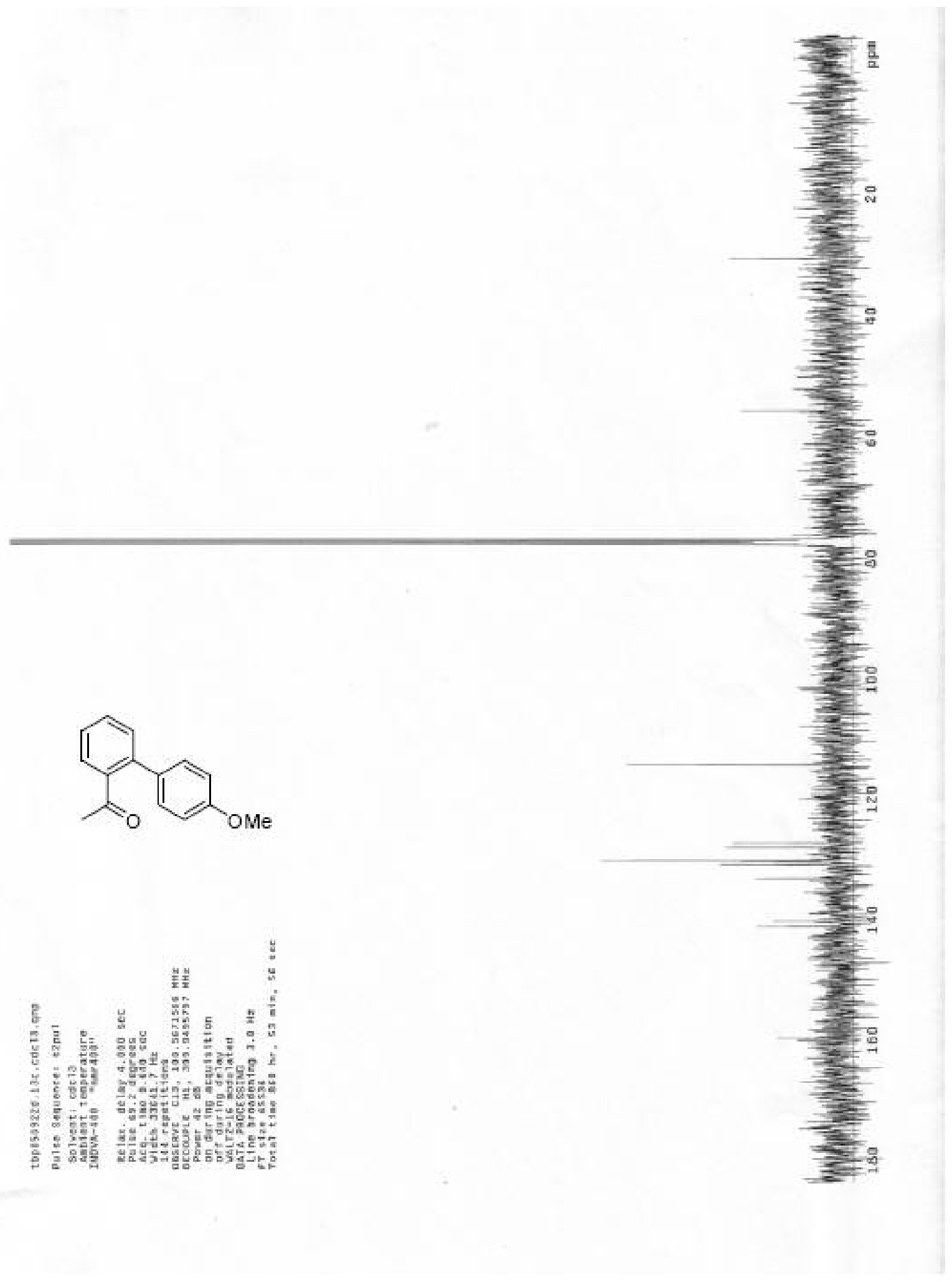

S-28 


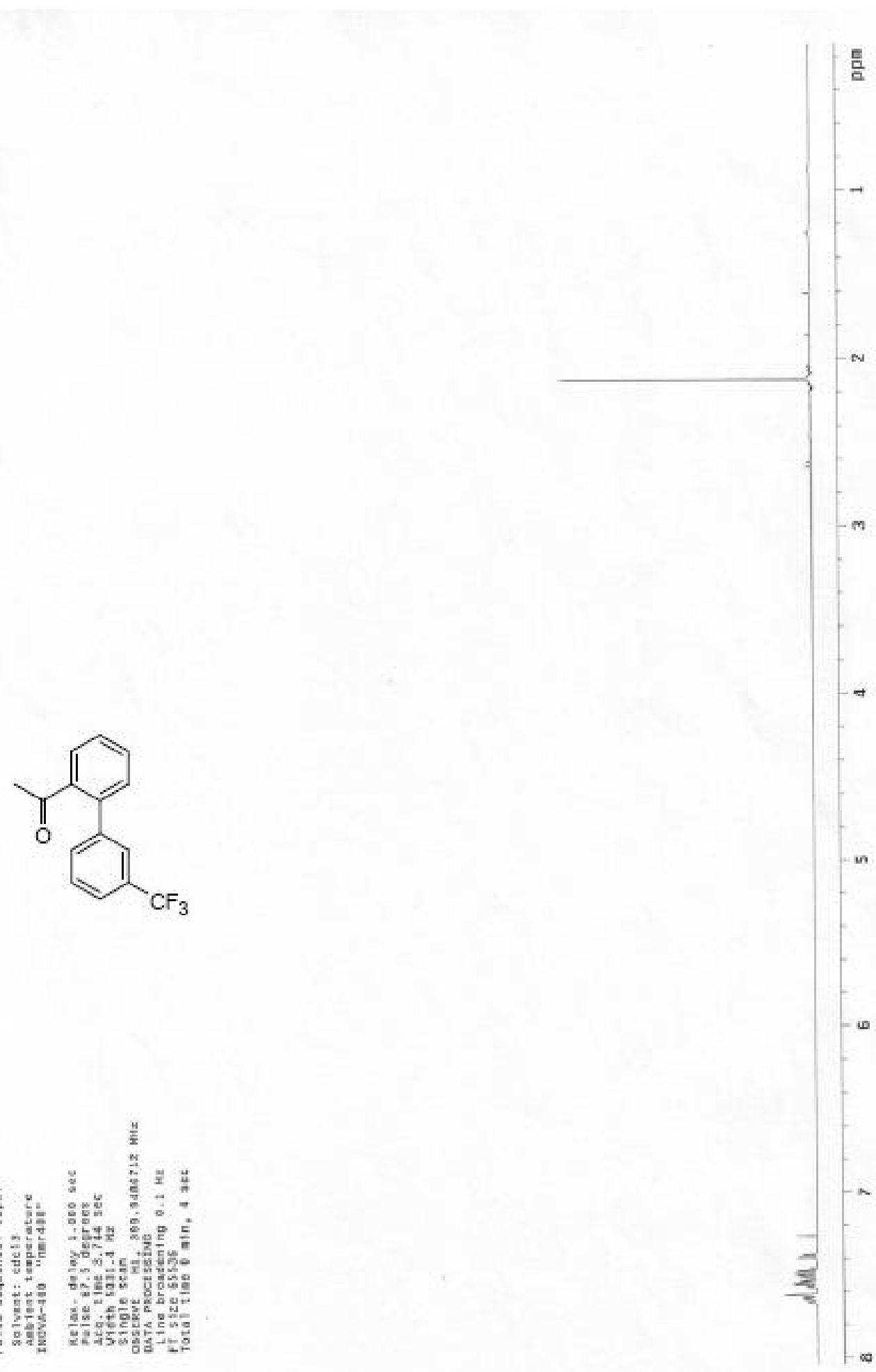



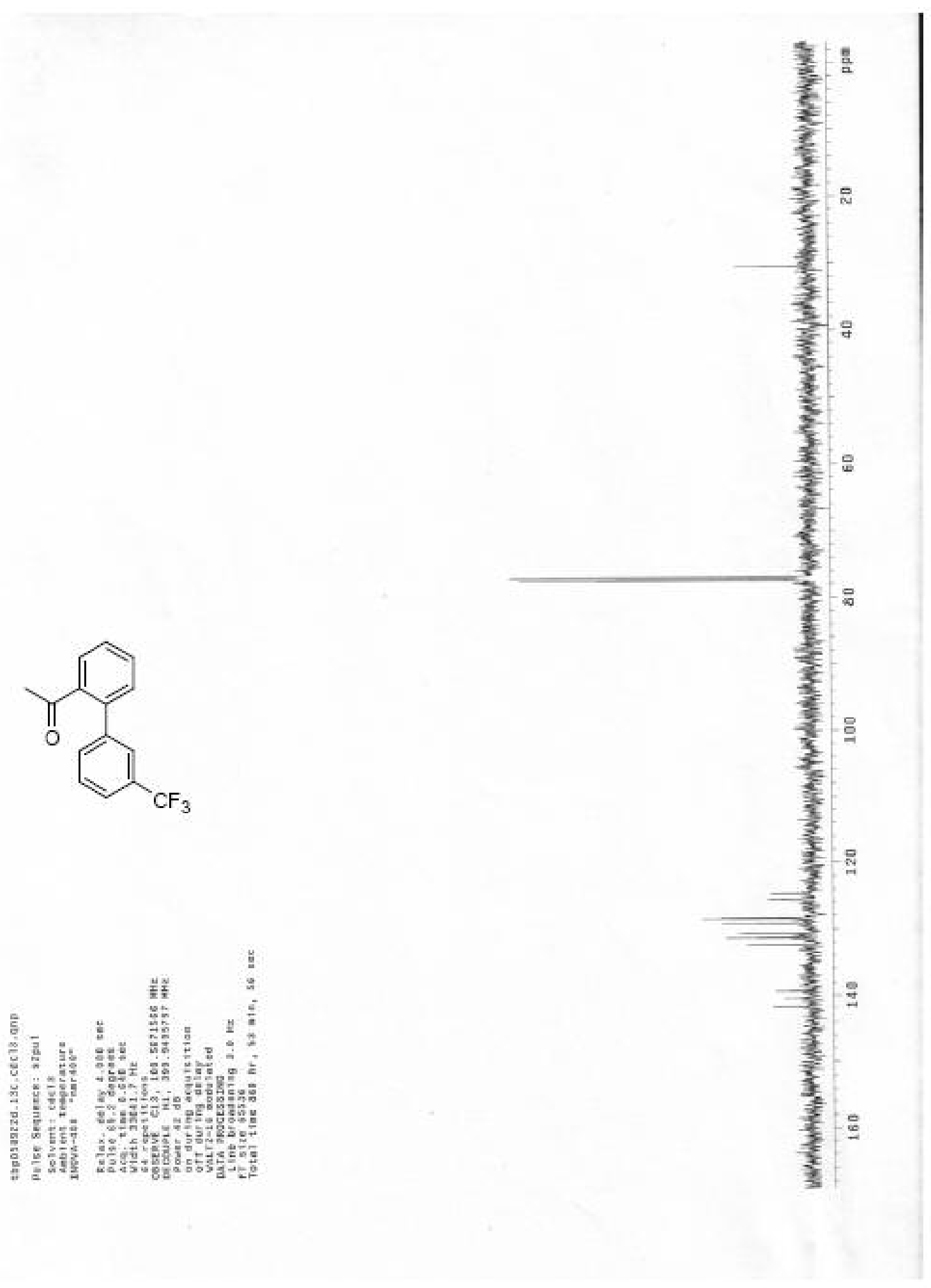

S-30 

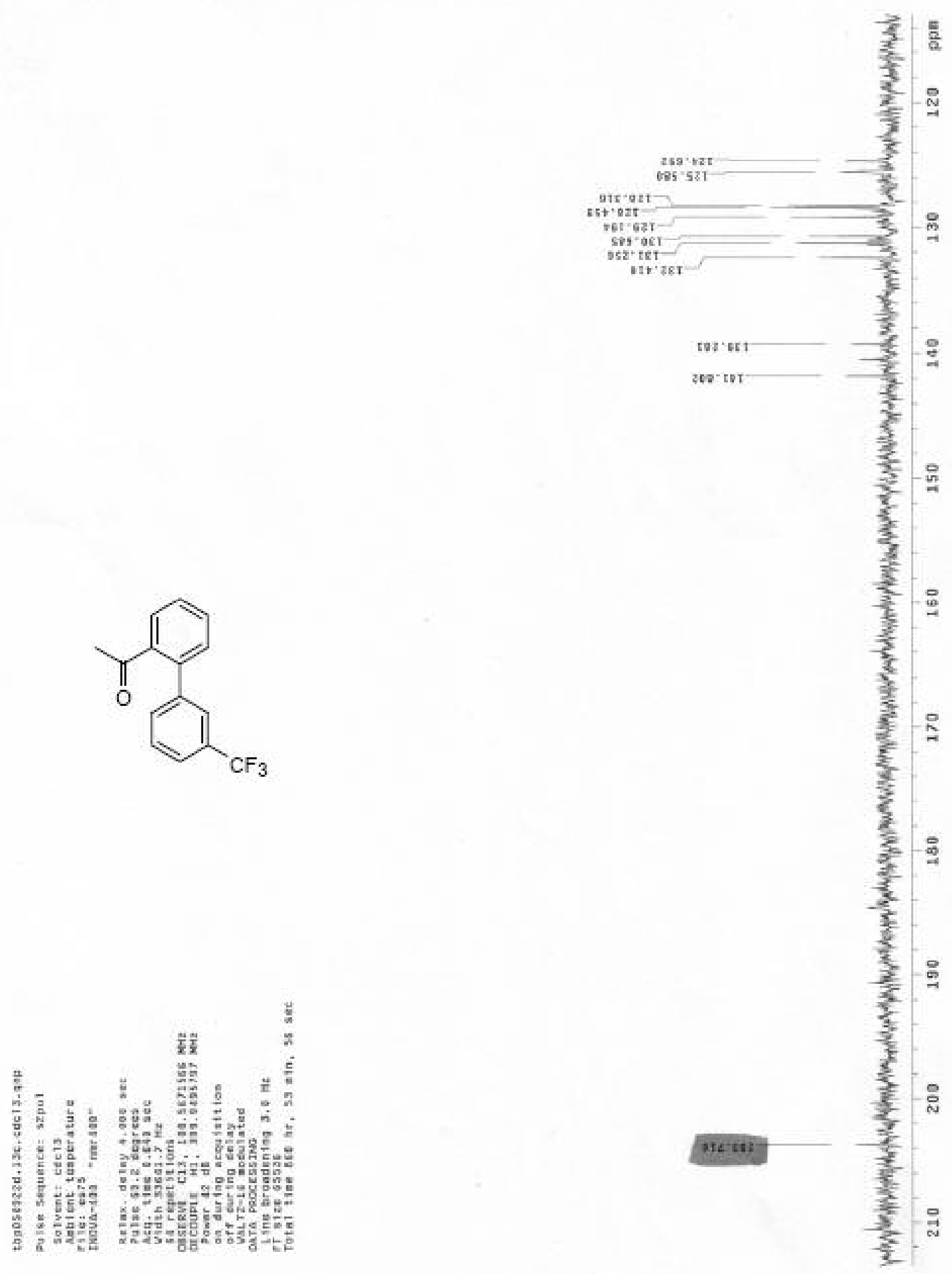


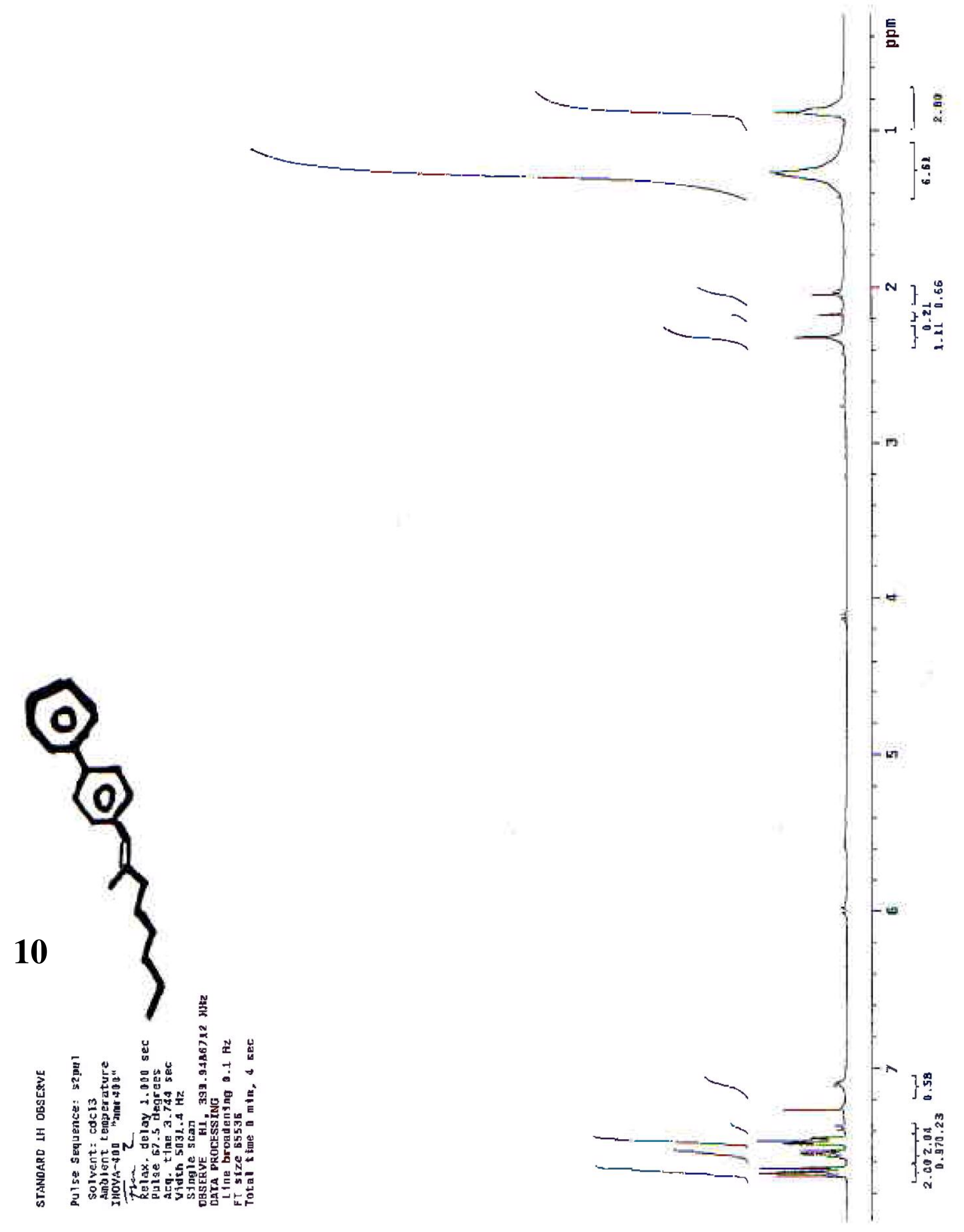




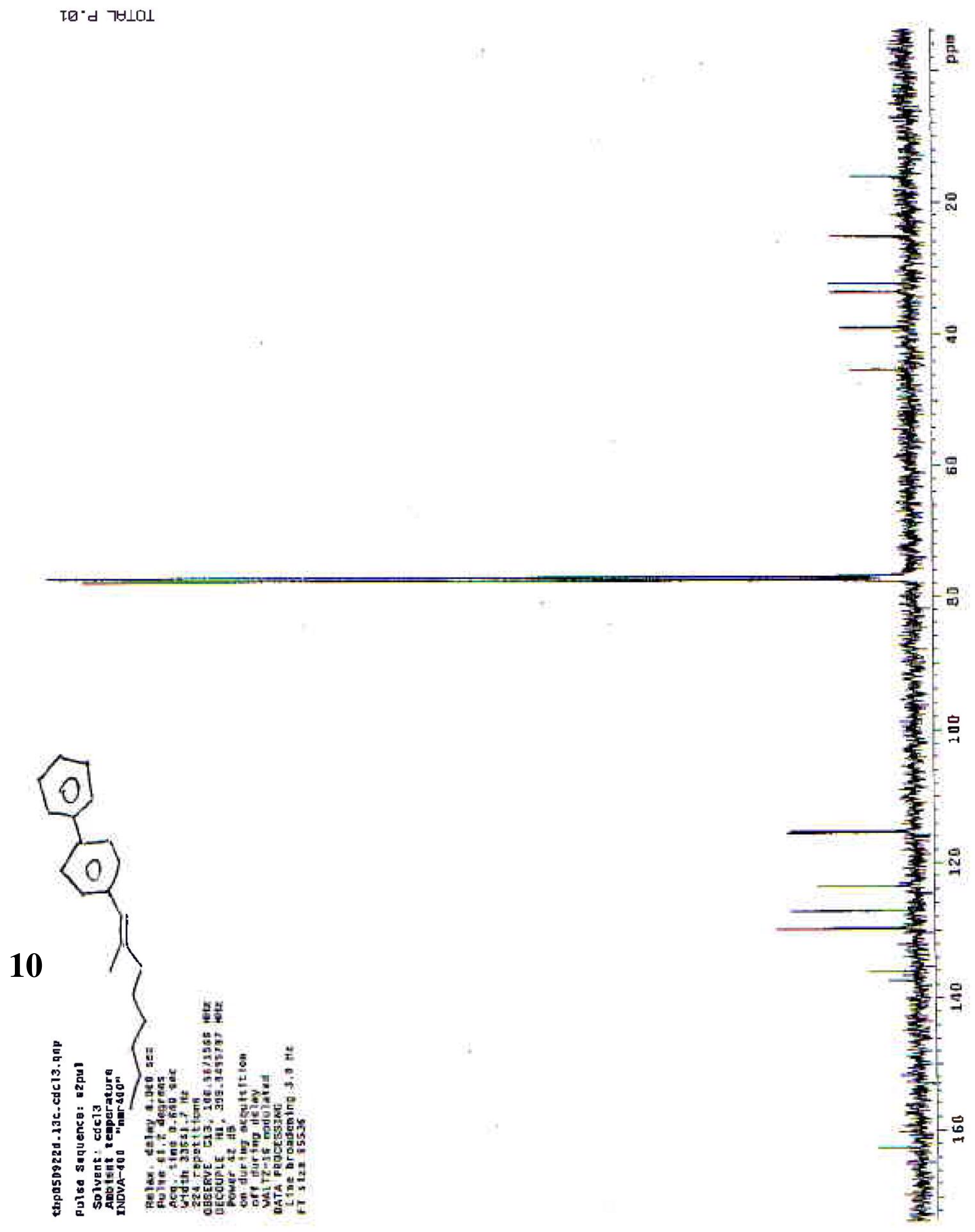



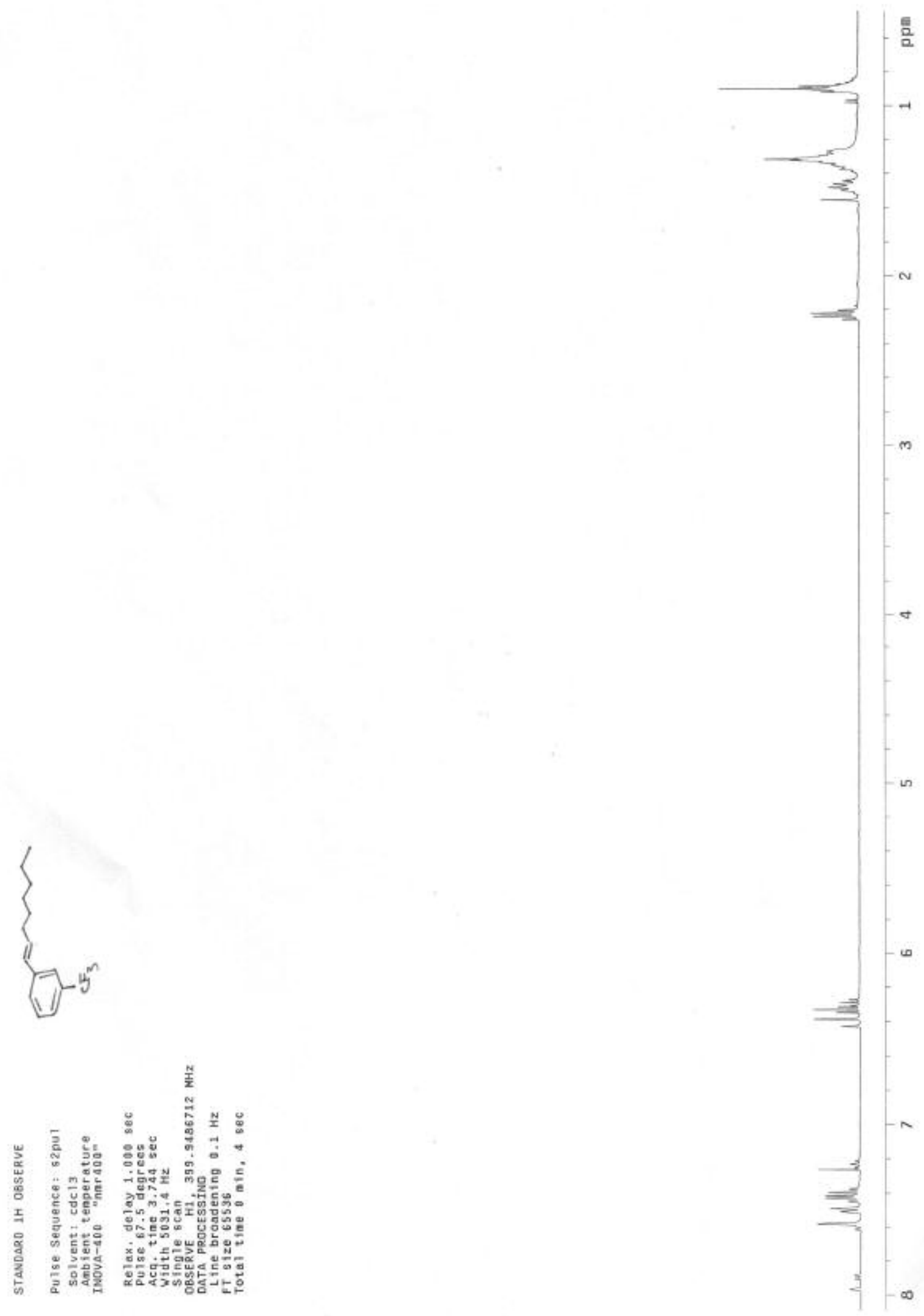


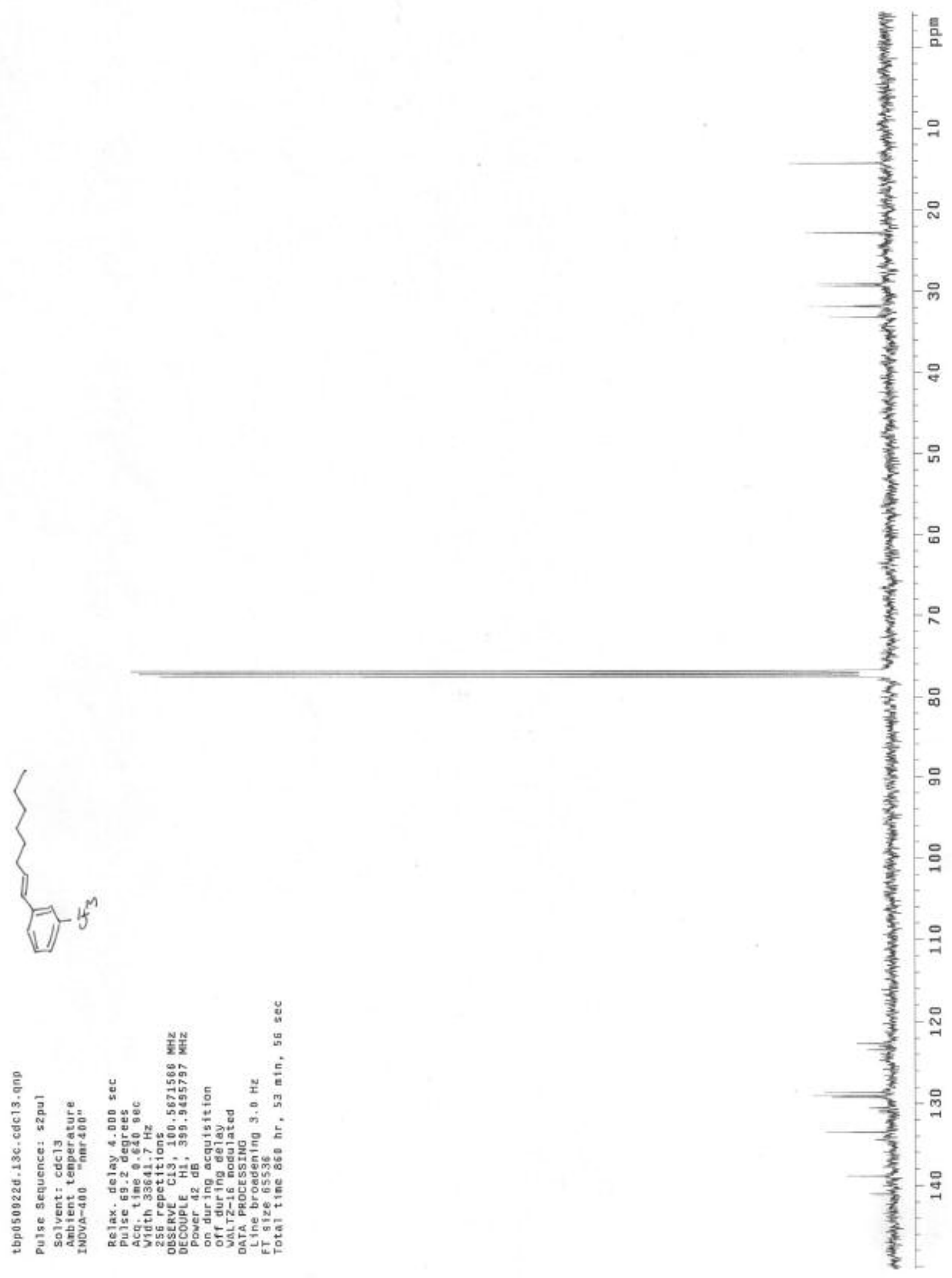

S-35 


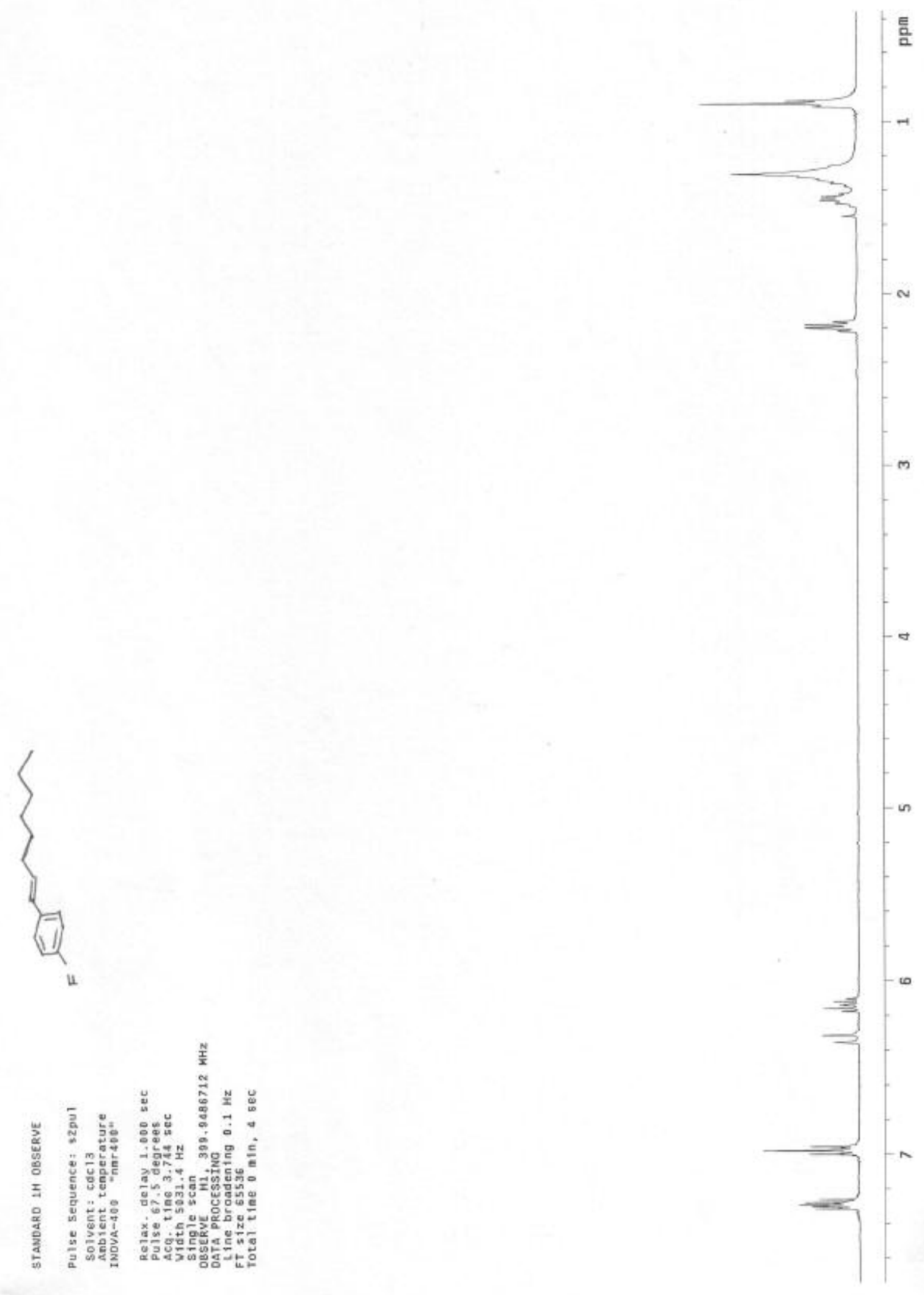

S-36 


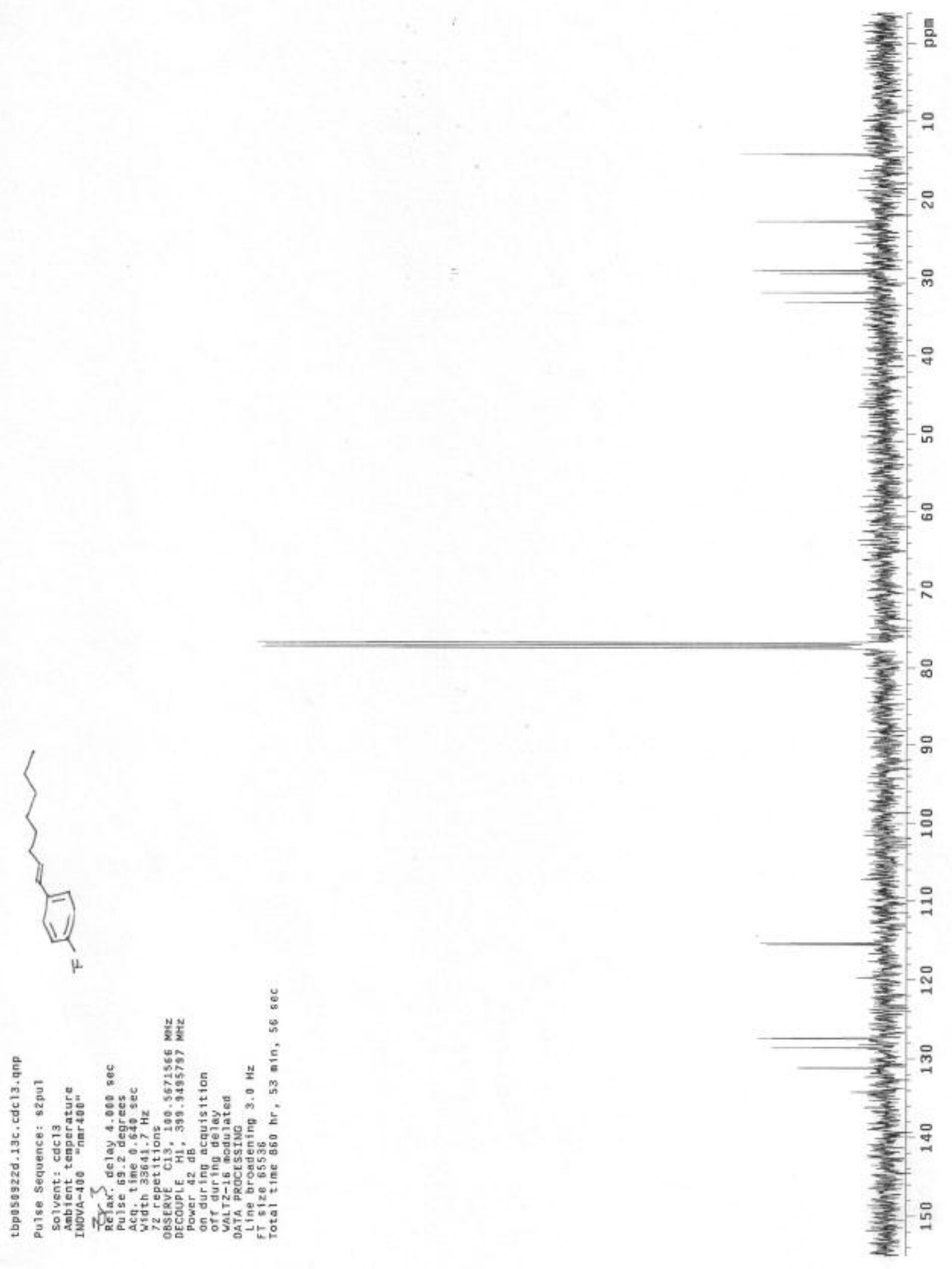

